\title{
Multiple Existence of Nonradial Positive Solutions for a Coupled Nonlinear Schrödinger System
}

Norimichi Hirano

\begin{abstract}
In this paper, we consider the multiple existence of nonradial positive solutions of coupled nonlinear Schrödinger system

$$
\left\{\begin{array}{l}
-\Delta u+\mu_{1} u=u^{3}+\beta v^{2} u \quad \text { in } \mathbb{R}^{3} \\
-\Delta v+\mu_{2} v=v^{3}+\beta u^{2} v \quad \text { in } \mathbb{R}^{3}
\end{array}\right.
$$

where $\mu_{1}, \mu_{2}>0$ with $\mu_{1} \neq \mu_{2}$ and $\beta<0$.

It is known that the solutions of $(\mathrm{P})$ is not necessarily radial [12]. We show that problem $(\mathrm{P})$ has multiple nonradial solutions in case that $|\beta|$ is sufficiently small.
\end{abstract}

Mathematics Subject Classification (2000). Primary 35B40, 35B45; Secondary $35 \mathrm{~J} 40$.

Keywords. Coupled Schrődinger equations, sign changing solutions.

\section{Introduction}

In the present paper, we consider the multiple existence of nonradial positive solutions of coupled Schrödinger system

$$
\left\{\begin{array}{l}
-\Delta u+\mu_{1} u=u^{3}+\beta u v^{2} \quad \text { in } \quad \mathbb{R}^{3} \\
-\Delta v+\mu_{2} v=v^{3}+\beta u^{2} v \quad \text { in } \quad \mathbb{R}^{3}
\end{array}\right.
$$

where $\mu_{1}, \mu_{2}>0$ and $\beta<0$.

Coupled nonlinear Schrödinger system $(\mathrm{P})$ models many physical problems. In nonlinear optics, the phenomenon in Kerr-like photorefractive media is described by system (P) (cf. [2]). In this case, the solution $u$ and $v$ denote the components of the beam in Kerr-like photorefractive media, and the coupling constant $\beta$ is the interaction between two components $u$ and $v$. In case $\beta>0$, the interaction is attractive, while the interaction is repulsive if $\beta<0$. The bimodal pulse in 
optical fibers under birefringent effects is also governed by system (P) (cf. [15]). System $(\mathrm{P})$ is also a model for a mixture of two Bose-Einstein condensates (cf. [6]).

Recently motivated by these physical interest, the existence of solutions of $(\mathrm{P})$ has been investigated by several authors. In the case that $\beta>0$, problem $(\mathrm{P})$ was studied by Ambrosetti \& Colorado [3], Maia, Montefusco \& Pellacci [14] and Lin $\&$ Wei $[10]$. They proved the existence of least energy solutions $(u, v)$ of $(\mathrm{P})$ with $u, v>0$. We note that in this case, all positive solutions $(u, v)$ of $(\mathrm{P})$ satisfying

$$
u(x) \longrightarrow 0, \quad v(x) \longrightarrow 0, \text { as }|x| \longrightarrow \infty
$$

are radially symmetric functions(cf. [19]). On the other hand, in case that $\beta<0$, it is known that there is no least energy solution of $(\mathrm{P})$ (cf. [10]). Moreover, positive solutions of $(\mathrm{P})$ satisfying (1.1) is not always radial. In case that $\beta<0$ and $|\beta|$ is small, there are positive solutions with one component concentrating on the origin and the other component concentrating around a regular polygon(cf. [12]). The existence of non radial positive solutions was also considered in [21] for the case that $\beta<0$ and $\mu_{1}=\mu_{2}$. In this case, there are infinitely many nonradial solutions if $\beta<-1$. The existence of sign changing solutions of $(\mathrm{P})$ with $\beta>0$ was considered in $[8]$. For the problem $(\mathrm{P})$ with $\mathbb{R}^{3}$ replaced by a bounded domain, we refer to [11] and [16].

In the present paper, we are interested in the case that $\beta<0$ and $|\beta|$ is small, i.e. the case that the interaction of two solutions are small and repulsive. We will show the multiple existence of nonradial solutions of $(\mathrm{P})$ in this case with $\mu_{1} \neq \mu_{2}$. Our results improve the results in [12].(See Remark 1 and Remark 3).

To state our main results, we need some notations. We denote by $B_{r}(x)$ the open ball in $\mathbb{R}^{3}$ centered at $x \in \mathbb{R}^{3}$ with radius $r>0$. The inner product in $\mathbb{R}^{3}$ is denoted by $\langle\cdot, \cdot\rangle_{\mathbb{R}^{3}}$. We put $H=H^{1}\left(\mathbb{R}^{3}\right)$ and $\mathbb{H}=H \times H$. We set $\mu_{0}=1$. We denote by $\|\cdot\|_{\mu_{i}}$ the norm of $H$ defined by $\|u\|_{\mu_{i}}^{2}=\int_{\mathbb{R}^{3}}\left(|\nabla u|^{2}+\mu_{i}|u|^{2}\right) d x$ for $u \in H$ and $i \in\{0,1,2\}$. For simplicity of notations, we put $|u(x)|_{\mu_{i}}^{2}=|\nabla u(x)|^{2}+$ $\mu_{1}|u(x)|^{2}$ for $u \in H$ and $x \in \mathbb{R}^{3}$. For each function $u \in H$, we set $u^{+}(x)=$ $\max \{u(x), 0\}, u^{-}(x)=\max \{-u(x), 0\}$. For each $p \geq 1$, we denote by $|\cdot|_{p}$ the norm of the space $L^{p}\left(\mathbb{R}^{3}\right)$. The Hilbert space $\mathbb{H}$ is equipped with the norm defined by $\|\mathcal{U}\|^{2}=\|u\|_{\mu_{1}}^{2}+\|v\|_{\mu_{2}}^{2}$ for $\mathcal{U}=(u, v) \in \mathbb{H}$. We recall that for each $i \in\{0,1,2\}$, problem

$$
\left\{\begin{array}{l}
-\Delta u+\mu_{i} u=u^{3} \text { in } \mathbb{R}^{3} \\
u(x)>0 \text { in } \mathbb{R}^{3} \\
u(x) \longrightarrow 0 \text { as }|x| \longrightarrow \infty
\end{array}\right.
$$

has a radial solution, denoted by $U_{i}$ (cf. $\left.[7,9]\right)$. The function $U_{i}$ is the unique smooth solution of $\left(\mathrm{P}_{i}\right)$ up to translation. Moreover we know that $U_{i}$ satisfies $I_{i}\left(U_{i}\right)=c_{i}=$ $\min \left\{I(v): v \in \mathcal{S}_{i}\right\}$, where $I_{i}$ is the functional associated with problem $\left(P_{i}\right)$ defined by

$$
I_{i}(v)=\frac{1}{2}\|v\|_{\mu_{i}}^{2}-\frac{1}{4}\left|v^{+}\right|_{4}^{4} \quad \text { for } \quad v \in H
$$


and $\mathcal{S}_{i}$ is the set defined by

$$
\mathcal{S}_{i}=\left\{v \in H:\|v\|_{\mu_{i}}^{2}=\left|v^{+}\right|_{4}^{4}\right\} \quad \text { for } \quad i=1,2 .
$$

For each $x \in \mathbb{R}^{3}$ and $i \in\{0,1,2\}$, we put $U_{i, x}(\cdot)=U_{i}(\cdot-x)$. It is also known that

$$
\left|U_{i}(x)\right|_{\mu_{i}}|x| \exp \left(\sqrt{\mu_{i}}|x|\right) \longrightarrow c>0, \quad \text { as } \quad|x| \longrightarrow \infty \text { for } i \in\{0,1,2\} .
$$

(cf. [9]). For each $u \in L^{4}\left(\mathbb{R}^{3}\right)$, we put $\widehat{u}(x)=\int_{B_{1}(x)}|u(x)|^{4} d x$ for $x \in \mathbb{R}^{3}$. Then from (1.3), we can choose $R_{0}>0$ such that

$$
\widehat{U_{i}}(z)<\frac{\left|\widehat{U}_{i}\right|_{\infty}}{3} \quad \text { for all } \quad z \in \mathbb{R}^{3} \backslash B_{R_{0}}(0) \text { and } i \in\{1,2\} .
$$

Since we consider the case that $\mu_{1} \neq \mu_{2}$, we may assume without any loss of generality that $\mu_{2}<\mu_{1}$. We can now state our main results.

Theorem 1. Suppose that $\sqrt{\mu_{1} / \mu_{2}}$ is irrational. Then for each $i \in\{2,4,6,8,12,20\}$ there exists $\beta_{i} \in(-1,0)$ such that for each $\beta \in\left(\beta_{i}, 0\right)$, there exists a positive solution $\mathcal{U}_{i} \in \mathbb{H}$ of $(P)$ such that $i c_{1}+c_{2}<\Phi\left(\mathcal{U}_{i}\right)<i c_{1}+2 c_{2}$ and $\mathcal{U}_{i}$ has the form

$$
\mathcal{U}_{i}=(U, V)=\left(\sum_{j=1}^{i} U_{1, x_{j}}+u, U_{2}+v\right)
$$

where $\left\{x_{1}, x_{2}, \ldots, x_{i}\right\}$ forms a regular $i$-polyhedra in $\mathbb{R}^{3}$ in case $i \neq 2$ and $x_{1}=$ $-x_{2}$ in case that $i=2$, and $u, v \in H$ such that $\|(u, v)\|$ is so small that

$$
\begin{aligned}
\widehat{U}(z) & <\frac{1}{2}|\widehat{U}|_{\infty} \quad \text { for } \quad z \in \mathbb{R}^{3} \backslash\left(\cup_{j=1}^{i} B_{R_{0}}\left(x_{j}\right)\right) \quad \text { and } \\
\widehat{V}(z) & <\frac{1}{2}|\widehat{V}|_{\infty} \quad \text { for } \quad z \in \mathbb{R}^{3} \backslash B_{R_{0}}(0) .
\end{aligned}
$$

Remark 1. The assertion of Theorem 1 implies that for $\beta<0$ with $|\beta|$ sufficiently small, problem $(P)$ possesses at least 6 nonradial positive solutions. In [12], the existence of positive solutions of $(P)$ of the form (1.5) was established in the case that $\left\{x_{1}, x_{2}, \ldots, x_{i}\right\}$ forms regular cube or tetrahedra under the assumption

$$
\sqrt{\frac{\mu_{1}}{\mu_{2}}}< \begin{cases}\frac{\sqrt{3}}{3} & \text { for the cube } \\ \frac{\sqrt{3}}{2} & \text { for the tetrahedra. }\end{cases}
$$

Our argument employed in this paper does not require the ratio of $\sqrt{\mu_{1}}$ and $\sqrt{\mu_{2}}$.

Remark 2. One can see, from the proof of Theorem 1, that under condition (1.6), the absolute value $\left|x_{i}\right|$ of $x_{i}$ is uniquely determined.

Theorem 2. Suppose that $\sqrt{\mu_{1} / \mu_{2}}$ is irrational. Then for each $k \in \mathbb{N}$, there exists $\widetilde{\beta}_{k} \in(-1,0)$ such that for each $\beta \in\left(\widetilde{\beta}_{k}, 0\right)$, the problem $(P)$ has a positive solution 
$\widetilde{\mathcal{U}}_{k}$ such that $k c_{1}+c_{2}<\Phi\left(\widetilde{\mathcal{U}}_{k}\right)<k c_{1}+2 c_{2}$ and $\widetilde{\mathcal{U}}_{k}$ has the form

$$
\widetilde{\mathcal{U}}_{k}=(U, V)=\left(\sum_{j=1}^{k} U_{1, x_{j}}+u, U_{2}+v\right)
$$

where $\left\{x_{1}, x_{2}, \ldots, x_{k}\right\} \subset \mathbb{R}^{3}$ form a regular $k$-polygon in a two dimensional subspace of $\mathbb{R}^{3}$, and $u, v \in H$ such that $\|(u, v)\|$ is so small that

$$
\widehat{U}(z)<\frac{1}{2}|\widehat{U}|_{\infty} \quad \text { for } \quad z \in \mathbb{R}^{3} \backslash\left(\cup_{j=1}^{k} B_{R_{0}}\left(x_{j}\right)\right)
$$

and

$$
\widehat{V}(z)<\frac{1}{2}|\widehat{V}|_{\infty} \quad \text { for } \quad z \in \mathbb{R}^{3} \backslash B_{R_{0}}(0) .
$$

Remark 3. The existence of positive solutions of $(P)$ of the form (1.7) was proved in [12] in the case that the spacial dimension is 2 and $\mu_{1}, \mu_{2}$ satisfy

$$
\sqrt{\frac{\mu_{1}}{\mu_{2}}}<\sin \frac{\pi}{k}
$$

To avoid unnecessary complexity, we give a proof of Theorem 1 for the case $i=2$ in Section 2 and Section 3. The proofs of Theorem 1 for $i \neq 2$ and the proof of Theorem 2 are slight modifications of that of the case $i=2$ of Theorem 1 . The sketch of proofs for these cases are given in Section 4.

\section{Preliminaries}

Throughout the rest of this paper, we assume that $\sqrt{\mu_{1} / \mu_{2}}$ is irrational. For each $u, v \in H=H^{1}\left(\mathbb{R}^{3}\right)$, we put $\langle u, v\rangle=\int_{\mathbb{R}^{3}} u v$. We denote by $\langle\cdot, \cdot\rangle_{\mu_{i}}$ the inner product of $H$ defined by $\langle u, v\rangle_{\mu_{i}}=\int_{\mathbb{R}^{3}}\left(\nabla u \cdot \nabla v+\mu_{i} u v\right)$ for $u, v \in H$ and $i \in$ $\{0,1,2\}$. The inner product of $\mathbb{H}$ is defined by $\left\langle\mathcal{U}_{1}, \mathcal{U}_{2}\right\rangle_{\mathbb{H}}=\left\langle U_{1}, U_{2}\right\rangle_{\mu_{1}}+\left\langle V_{1}, V_{2}\right\rangle_{\mu_{2}}$ for $\mathcal{U}_{1}=\left(U_{1}, V_{1}\right), \mathcal{U}_{2}=\left(U_{2}, V_{2}\right) \in \mathbb{H}$. For each $u \in L^{4}\left(\mathbb{R}^{3}\right)$, we put $\Omega(u)=\{x \in$ $\left.\mathbb{R}^{3}: \widehat{u}(x) \geq \frac{|\widehat{u}|_{\infty}}{2}\right\}$ and

$$
\mathcal{B}(u)=\frac{\int_{\Omega(u)} x\left(\widehat{u}(x)-\frac{|\widehat{u}|_{\infty}}{2}\right) d x}{\int_{\Omega(u)}\left(\widehat{u}(x)-\frac{|\widehat{u}|_{\infty}}{2}\right) d x} .
$$

The mapping $\mathcal{B}$ is called generalized barycenter, which is introduced in [5] (cf. also [4]). By Sobolev's embedding theorem [1], for $p \in[2,6]$ there exists $m_{p}>0$ such that

$$
|z|_{L^{p}\left(B_{r}(0)\right)} \leq m_{p}\left(|\nabla z|_{L^{2}\left(B_{r}(0)\right)}^{2}+\mu_{i}|z|_{L^{2}\left(B_{r}(0)\right)}^{2}\right)^{1 / 2}
$$

for $r>1, z \in H^{1}\left(B_{r}(0)\right)$, and $i \in\{0,1,2\}$. For each $a \in \mathbb{R}$, and a functional $F: \mathbb{H}$ $\rightarrow \mathbb{R}$, we denote by $F^{a}$ the level set defined by $F^{a}=\left\{v \in \mathbb{H}: F^{a}(v) \leq a\right\}$. The same notation is used for functionals defined on $H$. 
It is easy to see that for each $u \in H \backslash\{0\}$ with $u^{+} \not \equiv 0$, there exists a unique positive number $t$ such that $t u \in \mathcal{S}_{i}$ (cf. [22]). It follows from the definitions of $U_{i}$ that $U_{i}(x)=\sqrt{\mu_{i}} U_{0}\left(\sqrt{\mu_{i}} x\right)$ on $\mathbb{R}^{3}$. Then one can see that

$$
c_{1}=\sqrt{\mu_{1}} c_{0}>c_{2}=\sqrt{\mu_{2}} c_{0} .
$$

Let $i \in\{0,1,2\}$. It is known that $\left\{U_{i, x}: x \in \mathbb{R}^{3}\right\}$ is a nondegenerate critical set of $I_{i}$ (cf. [20]). More precisely, we have there exists $\lambda>0$ such that

$$
\|u\|_{\mu_{i}}^{2}-3\left\langle U_{i}^{2} u, u\right\rangle \geq \lambda\|u\|_{\mu_{i}}^{2} \quad \text { for all } \quad u \in\left\{U_{i}, \frac{\partial U_{i}}{\partial x_{1}}, \frac{\partial U_{i}}{\partial x_{2}}, \frac{\partial U_{i}}{\partial x_{3}}\right\}^{\perp} .
$$

We define a functional $\Phi: \mathbb{H} \longrightarrow \mathbb{R}$ associated with problem $(\mathrm{P})$ by

$$
\begin{aligned}
\Phi(\mathcal{U}) & =\frac{1}{2}\left(\|U\|_{\mu_{1}}^{2}+\|V\|_{\mu_{2}}^{2}\right)-\frac{1}{4}\left(\left|U^{+}\right|_{4}^{4}+\left|v^{+}\right|_{4}^{4}\right)-\frac{\beta}{2} \int_{\mathbb{R}^{3}}\left(U^{+}\right)^{2}\left(V^{+}\right)^{2} \\
& =\Phi_{1}(\mathcal{U})+\Phi_{2}(\mathcal{U}) \quad \text { for } \quad \mathcal{U}=(U, V) \in \mathbb{H}
\end{aligned}
$$

where

$$
\Phi_{1}(\mathcal{U})=\frac{1}{2}\|U\|_{\mu_{1}}^{2}-\frac{1}{4}\left|U^{+}\right|_{4}^{4}-\frac{\beta}{4} \int_{R^{3}}\left(U^{+}\right)^{2}\left(V^{+}\right)^{2}
$$

and

$$
\Phi_{2}(\mathcal{U})=\frac{1}{2}\|V\|_{\mu_{1}}^{2}-\frac{1}{4}\left|v^{+}\right|_{4}^{4}-\frac{\beta}{4} \int_{R^{3}}\left(U^{+}\right)^{2}\left(V^{+}\right)^{2}
$$

Then a direct computation shows

$$
\begin{aligned}
\langle\nabla \Phi(\mathcal{U}), \mathcal{V}\rangle_{\mathbb{H}}= & \left\langle\left(\begin{array}{c}
\nabla_{u} \Phi(\mathcal{U}) \\
\nabla_{v} \Phi(\mathcal{U})
\end{array}\right),\left(\begin{array}{c}
W \\
Z
\end{array}\right)\right\rangle_{\mathbb{H}} \\
= & \left\langle-\Delta U+\mu_{1} U-\left(U^{+}\right)^{3}-\beta U^{+}\left(V^{+}\right)^{2}, W\right\rangle \\
& +\left\langle-\Delta V+\mu_{2} V-\left(V^{+}\right)^{3}-\beta\left(U^{+}\right)^{2} V^{+}, Z\right\rangle
\end{aligned}
$$

for $\mathcal{U}=(U, V), \mathcal{V}=(W, Z) \in \mathbb{H}$. We put

$$
\begin{aligned}
\mathcal{M}_{+} & =\left\{(U, V) \in \mathbb{H} \backslash\{0\}:\|U\|_{\mu_{1}}^{2}=\left|U^{+}\right|_{4}^{4}+\beta \int_{\mathbb{R}^{3}}\left(U^{+}\right)^{2}\left(V^{+}\right)^{2},\right. \\
\|V\|_{\mu_{2}}^{2} & \left.=\left|V^{+}\right|_{4}^{4}+\beta \int_{\mathbb{R}^{3}}\left(U^{+}\right)^{2}\left(V^{+}\right)^{2}\right\} .
\end{aligned}
$$

Then one can see that $\mathcal{U}=(U, V) \in \mathcal{M}_{+}$is a critical point of $\Phi$ if and only if $\mathcal{U}$ is a positive solution of problem $(\mathrm{P})$. From the definition, we have

$$
\Phi_{1}(\mathcal{U})=\frac{1}{4}\|U\|_{\mu_{1}}^{2}, \quad \Phi_{2}(\mathcal{U})=\frac{1}{4}\|V\|_{\mu_{2}}^{2} \quad \text { and } \quad \Phi(\mathcal{U})=\frac{1}{4}\left(\|U\|_{\mu_{1}}^{2}+\|V\|_{\mu_{2}}^{2}\right)
$$

for $\mathcal{U}=(U, V) \in \mathcal{M}_{+}$. We also have that for each $\mathcal{U}=(U, V) \in \mathcal{M}_{+}$, there exists $(s, t) \in \mathbb{R}^{+} \times \mathbb{R}^{+}$such that $(s U, t V) \in \mathcal{M}_{+}$. In fact, for each $\mathcal{U}=(U, V)$, $U \not \equiv 0, V \not \equiv 0,(s U, t V) \in \mathcal{M}_{+}$if and only if

$$
\left(\begin{array}{c}
s^{2} \\
t^{2}
\end{array}\right)=A^{-1}\left(\begin{array}{c}
\|U\|_{\mu_{1}}^{2} \\
\|V\|_{\mu_{2}}^{2}
\end{array}\right)
$$


where

$$
A=\left(\begin{array}{cc}
\left|U^{+}\right|_{4}^{4} & \beta \int_{\mathbb{R}^{3}}\left(U^{+}\right)^{2}\left(V^{+}\right)^{2} \\
\beta \int_{\mathbb{R}^{3}}\left(U^{+}\right)^{2}\left(V^{+}\right)^{2} & \left|V^{+}\right|_{4}^{4}
\end{array}\right) .
$$

Since $\beta \in(-1,0)$, we have by the Schwartz's inequality that $A^{-1}$ exists and then there exists a unique solution $(s, t) \in \mathbb{R}^{+} \times \mathbb{R}^{+}$. For given $\mathcal{U}=(U, V) \in \mathbb{H}$ with $U \not \equiv 0, V \not \equiv 0$, we put $\mathcal{N} \mathcal{U}=\mathcal{N}(U, V)=\left(\mathcal{N}_{1} U, \mathcal{N}_{2} V\right)=(s U, t V) \in \mathcal{M}_{+}$.

We start from the following lemma which is a simple consequence from the uniqueness of the positive solution of $\left(P_{i}\right)$

Lemma 1. Let $i \in\{1,2\}$ and $\left\{u_{n}\right\} \subset S_{i}$ such that $\lim _{n \rightarrow \infty} I_{i}\left(u_{n}\right)=c_{i}$. Then there exists $\left\{x_{n}\right\} \subset \mathbb{R}^{3},\left\{\alpha_{n}\right\} \subset \mathbb{R}^{+}$and $\left\{v_{n}\right\} \subset H$ such that $\lim _{n \longrightarrow \infty} \alpha_{n}=1$, $v_{n} \in\left\{U_{i, x_{n}}\right\}^{\perp}, \lim _{n} \longrightarrow \infty\left\|v_{n}\right\|_{\mu_{i}}=0$

$$
x_{n}=\mathcal{B}\left(u_{n}\right) \quad \text { and } \quad u_{n}=\alpha_{n} U_{i, x_{n}}+v_{n} \quad \text { for each } n \geq 1 .
$$

Proof. The assertion follows from a standard concentrate compactness argument (cf. Proposition 2.1 of [18]). Let $i \in\{1,2\}$ and let $\left\{u_{n}\right\}$ satisfy the assumption. Then since $\left\{u_{n}\right\} \subset S_{i}$ is a minimizing sequence for the functional $I_{i}$ on $S_{i}$, we have, by the uniqueness of the positive solution of $\left(P_{i}\right)$ up to translation, that there exists $\left\{\widetilde{x}_{n}\right\} \subset \mathbb{R}^{3},\left\{\alpha_{n}\right\} \subset \mathbb{R}^{+}$and $\left\{\widetilde{v}_{n}\right\} \subset H$ such that $\lim _{n \rightarrow \infty}\left\|\widetilde{v}_{n}\right\|=0$ and $u_{n}=\alpha_{n} U_{i, \widetilde{x}_{n}}+\widetilde{v}_{n}$ for $n \geq 1$. We may assume that $\alpha=1$. Then by the definition of $\mathcal{B}$, we have $\lim _{n} \longrightarrow \infty\left|\mathcal{B}\left(u_{n}\right)-\widetilde{x}_{n}\right|=0$. Then putting $x_{n}=\mathcal{B}\left(u_{n}\right)$, we find that $u_{n}=U_{i, x_{n}}+\left(U_{i, \widetilde{x}_{n}}-U_{i, x_{n}}+\widetilde{v}_{n}\right)$ for $n \geq 1$ and

$$
\lim _{n \longrightarrow \infty}\left\|U_{i, \widetilde{x}_{n}}-U_{i, x_{n}}+\widetilde{v}_{n}\right\|_{\mu_{i}}=0 .
$$

Let $\left\{\left(\theta_{n}, v_{n}\right)\right\} \subset \mathbb{R} \times H$ such that $v_{n} \in\left\{U_{i, x_{n}}\right\}^{\perp}$ and

$$
U_{i, \widetilde{x}_{n}}-U_{i, x_{n}}+\widetilde{v}_{n}=\theta_{n} U_{i, x_{n}}+v_{n} \quad \text { for } \quad n \geq 1 \text {. }
$$

Then

$$
u_{n}=\left(1+\theta_{n}\right) U_{i, x_{n}}+v_{n} \quad \text { for } \quad n \geq 1 \text {, }
$$

$\alpha_{n}=\left(1+\theta_{n}\right) \longrightarrow 1$ as $n \longrightarrow \infty$ and $\lim _{n \longrightarrow \infty}\left\|v_{n}\right\|_{\mu_{i}}=0$. This completes the proof.

Lemma 2. Let $U \in S_{1}$ such that $U=\alpha U_{1, x}+u$ where $\alpha \in\left(\frac{3}{4}, 2\right), x \in \mathbb{R}^{3}$ and $u \in\left\{U_{1, x}\right\}^{\perp}$ with $\|u\|_{\mu_{1}}$ sufficiently small. Then

$$
I_{1}(U) \geq c_{1}+\frac{1}{6}\|u\|_{\mu_{1}}^{2}
$$

Proof. Let $U=\alpha U_{1, x}+u$ satisfy the assumption. If $\alpha \geq 1$, then noting that $u \in\left\{U_{1, x}\right\}^{\perp}$,

$$
I_{1}(U)=\frac{1}{4}\left\|\alpha U_{1, x}+u\right\|_{\mu_{1}}^{2} \geq \frac{1}{4}\left(\left\|U_{1, x}\right\|_{\mu_{1}}^{2}+\|u\|_{\mu_{1}}^{2}\right) \geq c_{1}+\frac{1}{4}\|u\|_{\mu_{1}}^{2} .
$$

Then (2.4) holds. We next assume that $\alpha<1$. Since $U \in S_{1}$, we have

$$
\left\|\alpha U_{1, x}+u\right\|_{\mu_{1}}^{2}=\left(\left(\alpha U_{1, x}+u\right)^{+}\right)^{4} \leq\left(\alpha U_{1, x}+u\right)^{4} .
$$


Then noting that $\|u\|_{\mu_{1}}^{2} \geq 3 \int_{\mathbb{R}^{3}} U_{1, x}^{2} u^{2}$ by (2.2) and $u \in\left\{U_{1, x}\right\}^{\perp}$, we have

$$
\begin{aligned}
\left(\alpha^{2}-\alpha^{4}\right)\left\|U_{1, x}\right\|_{\mu_{1}}^{2}+\|u\|_{\mu_{1}}^{2} & =\int_{\mathbb{R}^{3}}\left(4 \alpha^{3} U_{1, x}^{3} u+6 \alpha^{2} U_{1, x}^{2} u^{2}\right)+o\left(\|u\|_{\mu_{1}}^{2}\right) \\
& =4 \alpha^{3}\left\langle\left(-\Delta+\mu_{1}\right) U_{1, x}, u\right\rangle+\int_{\mathbb{R}^{3}} 6 \alpha^{2} U_{1, x}^{2} u^{2}+o\left(\|u\|_{\mu_{1}}^{2}\right) \\
& \leq 2 \alpha^{2}\|u\|_{\mu_{1}}^{2}+o\left(\|u\|_{\mu_{1}}^{2}\right) .
\end{aligned}
$$

That is

$$
\alpha^{2}\left(1-\alpha^{2}\right)\left\|U_{1, x}\right\|_{\mu_{1}}^{2} \leq\left(2 \alpha^{2}-1\right)\|u\|_{\mu_{1}}^{2}+o\left(\|u\|_{\mu_{1}}^{2}\right) .
$$

Then by an elemental computation, we find that

$$
\alpha^{2} \geq \frac{\left\|U_{1, x}\right\|_{\mu_{1}}^{2}-\frac{2}{9}\|u\|_{\mu_{1}}^{2}}{\left\|U_{1, x}\right\|_{\mu_{1}}^{2}}
$$

for $\|u\|_{\mu_{1}}^{2}$ sufficiently small. Therefore

$$
\left\|\alpha U_{1, x}+u\right\|_{\mu_{1}}^{2} \geq\left\|U_{1, x}\right\|_{\mu_{1}}^{2}-\frac{2}{9}\|u\|_{\mu_{1}}^{2}+\|u\|_{\mu_{1}}^{2} \geq\left\|U_{1, x}\right\|_{\mu_{1}}^{2}+\frac{2}{3}\|v\|_{\mu_{1}}^{2}
$$

for $\|u\|_{\mu_{1}}^{2}$ sufficiently small. This completes the proof.

Lemma 3. (1) For each $\beta<0$ and $\mathcal{U}=(U, V) \in \mathcal{M}_{+}$,

$$
\Phi_{1}(U)=\frac{1}{4}\|U\|_{\mu_{1}}^{2}>c_{1} \quad \text { and } \quad \Phi_{2}(V)=\frac{1}{4}\|V\|_{\mu_{2}}^{2}>c_{2} .
$$

(2) Let $\left\{\mathcal{U}_{n}\right\}=\left\{\left(U_{n}, V_{n}\right)\right\} \subset \mathbb{H} \backslash\{0\}$ satisfy $\lim _{n \longrightarrow \infty} \nabla \Phi\left(U_{n}, V_{n}\right)=0$. Then

$$
\liminf _{n \longrightarrow \infty} \Phi_{1}\left(\mathcal{U}_{n}\right) \geq c_{1} \quad \text { if } \quad \liminf _{n \longrightarrow \infty}\left\|U_{n}\right\|_{\mu_{1}}^{2}>0
$$

and

$$
\liminf _{n \longrightarrow \infty} \Phi_{2}\left(\mathcal{U}_{n}\right) \geq c_{2} \quad \text { if } \quad \liminf _{n \longrightarrow \infty}\left\|V_{n}\right\|_{\mu_{2}}^{2}>0 .
$$

Proof. (1) Let $\beta<0$. Let $\mathcal{U}=(U, V) \in \mathcal{M}_{+}$. Then we have

$$
\|U\|_{\mu_{1}}^{2}=\left|U^{+}\right|_{4}^{4}+\beta \int_{\mathbb{R}^{3}}\left(U^{+}\right)^{2}\left(V^{+}\right)^{2} \leq\left|U^{+}\right|_{4}^{4} .
$$

Then there exists $t \in(0,1]$ such that $\|U\|_{\mu_{1}}^{2}=t^{2}\left|U^{+}\right|_{4}^{4}$. Since $t U \in S_{1}$, we have that

$$
c_{1} \leq I_{1}(t U)=\frac{t^{2}}{4}\|U\|_{\mu_{1}}^{2} \leq \frac{1}{4}\|U\|_{\mu_{1}}^{2} .
$$

If $\|U\|_{\mu_{1}}^{2} / 4=c_{1}$, we have that $t=1$ and $U=U_{1, x}$ for some $x \in \mathbb{R}^{3}$. Then since $\operatorname{supp} U_{1, x}=\mathbb{R}^{3}, \int_{\mathbb{R}^{3}} U^{2} V^{2} \neq 0$. This implies $\|U\|_{\mu_{1}}^{2}=\left|U^{+}\right|_{4}^{4}+\int_{\mathbb{R}^{3}} \beta\left(U^{+}\right)^{2}\left(V^{+}\right)^{2}<$ $\left|U^{+}\right|_{4}^{4}$ and then $t<1$. This is a contradiction. Therefore strict inequality $\frac{1}{4}\|U\|_{\mu_{1}}^{2}>$ $c_{1}$ holds. Similarly, we have $\frac{1}{4}\|V\|_{\mu_{2}}^{2}>c_{2}$. This completes the proof of (1). 
(2) Let $\left\{\mathcal{U}_{n}\right\}=\left\{\left(U_{n}, V_{n}\right)\right\} \subset \mathbb{H} \backslash\{0\}$ satisfy the assumption. Then we have

$$
\left\|U_{n}\right\|_{\mu_{1}}^{2}-\left|U_{n}^{+}\right|_{4}^{4}-\beta \int_{\mathbb{R}^{3}}\left(U_{n}^{+}\right)^{+}\left(V_{n}^{+}\right)^{2} \longrightarrow 0, \quad \text { as } \quad n \longrightarrow \infty \text {. }
$$

Then by the same argument as in the proof of (1), we have that $\liminf _{n \longrightarrow \infty} \Phi_{1}\left(\mathcal{U}_{n}\right) \geq c_{1}$ if $\liminf _{n \longrightarrow \infty}\left\|U_{n}\right\|_{\mu_{1}}^{2}>0$. We also have by the same argument that $\liminf _{n \longrightarrow \infty} \Phi_{2}\left(\mathcal{U}_{n}\right) \geq c_{2}$ if $\liminf _{n \longrightarrow \infty}\left\|V_{n}\right\|_{\mu_{2}}^{2}>0$.

Now to prove Theorem 1 for the case that $i=2$, we define $H_{2} \subset H, \mathbb{H}_{2} \subset \mathbb{H}$ and $\mathcal{M}_{2} \subset \mathcal{M}_{+}$by

$$
H_{2}=\left\{u \in H: u(x)=u(-x) \text { for } \quad x \in \mathbb{R}^{3}\right\}, \quad \mathbb{H}_{2}=H_{2} \times H_{2},
$$

and

$$
\mathcal{M}_{2}=\mathcal{M}_{+} \cap \mathbb{H}_{2} .
$$

Since $\sqrt{\mu_{1} / \mu_{2}}$ is irrational, we can choose $\delta_{2} \in\left(0, c_{2}\right)$ so small that

$$
c_{1}+k c_{2} \notin\left[2 c_{1}+c_{2}-\delta_{2}, 2 c_{1}+c_{2}+\delta_{2}\right] \text { for all } k \in \mathbb{N} .
$$

Lemma 4. (1) There exists $\beta_{1} \in(-1,0)$ such that for each $\beta \in\left(\beta_{1}, 0\right)$ and each critical point $\mathcal{U} \in \mathcal{M}_{2} \cap \Phi^{2 c_{1}+2 c_{2}}$ of $\Phi$,

$$
\Phi(\mathcal{U}) \in \cup_{i \geq 1, j \geq 1}\left[i c_{1}+j c_{2}-\delta_{2} / 2, i c_{1}+j c_{2}+\delta_{2} / 2\right] .
$$

(2) Let $\beta \in\left(\beta_{1}, 0\right)$ and $\left\{\mathcal{U}_{n}\right\} \subset \mathcal{M}_{2}$ such that $\lim _{n \longrightarrow \infty} \nabla \Phi\left(\mathcal{U}_{n}\right)=0$ and

$$
\lim _{n \longrightarrow \infty} \Phi\left(\mathcal{U}_{n}\right)=2 c_{1}+c_{2}+\varepsilon \quad \text { with } \quad \varepsilon \in\left(0, \delta_{2} / 2\right) \text {. }
$$

Then there exists a convergent subsequence $\left\{\mathcal{U}_{n_{i}}\right\} \subset\left\{\mathcal{U}_{n}\right\}$.

Proof. (1) Let $\left\{\beta_{n}\right\} \subset(-1,0)$ with $\lim _{n \longrightarrow \infty} \beta_{n}=0$ and $\left\{\mathcal{U}_{n}\right\}=\left\{\left(U_{n}, V_{n}\right)\right\} \subset \mathcal{M}_{2}$ such that $\lim _{n \longrightarrow \infty} \nabla \Phi_{u}\left(\mathcal{U}_{n}\right)=\lim _{n \longrightarrow \infty} \nabla \Phi_{v}\left(\mathcal{U}_{n}\right)=0$ and $\lim _{n \longrightarrow \infty} \Phi\left(\mathcal{U}_{n}\right) \leq$ $2 c_{1}+2 c_{2}$. Then we have $\lim _{n \longrightarrow \infty} \nabla I_{1}\left(U_{n}\right)=\lim _{n \longrightarrow \infty} \nabla I_{2}\left(V_{n}\right)=0$. Then by the uniqueness of the positive solution of $\left(P_{i}\right)$, we have by the concentrate compactness argument (cf. Proposition 2.1 of [18]) that there exists $k_{1} \in \mathbb{N}$ and sequences $\left\{x_{n, i}\right\} \subset \mathbb{R}^{3}, 1 \leq i \leq k_{1}$ such that $\lim _{n \longrightarrow \infty}\left|x_{n, i}-x_{n, j}\right|=\infty$ for $i \neq j$, and

$$
U_{n}-\sum_{i=1}^{k_{1}} U_{1, x_{n, i}} \longrightarrow 0 \quad \text { as } n \longrightarrow \infty \quad \text { in } H \text {. }
$$

That is we find $\lim _{n \rightarrow \infty} \Phi_{1}\left(\mathcal{U}_{n}\right)=\lim _{n \rightarrow \infty} I_{1}\left(U_{n}\right)=k_{1} c_{1}$. Similarly, we have that $\lim _{n \rightarrow \infty} \Phi_{2}\left(\mathcal{U}_{n}\right)=k_{2} c_{2}$ for some $k_{2} \geq 1$. Then the assertion follows.

(2) Let $\left\{\mathcal{U}_{n}\right\}=\left\{\left(U_{n}, V_{n}\right)\right\} \subset \mathcal{M}_{2}$ satisfy the assumption. Then by subtracting subsequences, we may assume that $\left(U_{n}, V_{n}\right)$ has the form

$$
U_{n}=U+u_{n}, V_{n}=V+v_{n},
$$

where

$$
\left(U_{n}, V_{n}\right) \longrightarrow(U, V) \in \mathbb{H}_{2} \quad \text { weakly in } \mathbb{H} \quad \text { and strongly in } \quad L^{4}\left(\mathbb{R}^{3}\right)
$$


and $\left\{\left(u_{n}, v_{n}\right)\right\} \subset \mathbb{H}_{2}$ is such that

$$
\left(u_{n}, v_{n}\right) \longrightarrow(0,0) \quad \text { weakly in } \mathbb{H} \text { and strongly in } L_{l o c}^{4}\left(\mathbb{R}^{3}\right) \text {. }
$$

From the definition, we have that $(U, V)$ is a solution of $(\mathrm{P})$, and

$$
\lim _{n \longrightarrow \infty} \nabla \Phi\left(u_{n}, V\right)=\lim _{n \longrightarrow \infty} \nabla \Phi\left(U, v_{n}\right)=\lim _{n \longrightarrow \infty} \nabla \Phi\left(u_{n}, v_{n}\right)=0 .
$$

Case 1. Assume that $U \not \equiv 0$ and $\liminf _{n \longrightarrow \infty}\left\|u_{n}\right\|>0$. In this case, we reaches to a contradiction by a standard concentrate compactness argument. For completeness, we give a proof. Since $(U, V) \in \mathcal{M}_{+}, \Phi_{1}(U, V) \geq c_{1}$. We first note that vanishing of $\left\{\left|u_{n}\right|_{\mu_{1}}^{2}\right\}$ does not happen. ( See Lemma 1.1 of [13]). In fact, if $\left\{\left|u_{n}\right|_{\mu_{1}}^{2}\right\}$ is vanishing, i.e., $\lim _{n \longrightarrow \infty} \sup _{y \in \mathbb{R}^{3}} \int_{B_{R}(y)}\left|u_{n}\right|_{\mu_{1}}^{2}=0$ for each $R>0$, then we have

$$
\lim _{n \longrightarrow \infty} \sup _{y \in \mathbb{R}^{3}} \frac{\int_{B_{R}(y)}\left|u_{n}^{+}\right|^{4}}{\int_{B_{R}(y)}\left|u_{n}\right|_{\mu_{1}}^{2}}=0 \text { for each } \quad R>0 .
$$

Then we find that

$$
\lim _{n \longrightarrow \infty} \frac{\left|u_{n}^{+}\right|_{4}^{4}-\beta \int_{\mathbb{R}^{3}}\left(u_{n}^{+}\right)^{2}\left(v_{n}^{+}\right)^{2}}{\left\|u_{n}\right\|_{\mu_{1}}^{2}} \leq 0 .
$$

On the other hand, we have by (2.8) that

$$
\lim _{n \longrightarrow \infty} \frac{\left|u_{n}^{+}\right|_{4}^{4}-\beta \int_{\mathbb{R}^{3}}\left(u_{n}^{+}\right)^{2}\left(v_{n}^{+}\right)^{2}}{\left\|u_{n}\right\|^{2}}=1 .
$$

This is a contradiction. Then vanishing of $\left\{\left|u_{n}\right|_{\mu_{1}}\right\}$ does not happen. On the other hand, we can see, noting that $u_{n}(x)=u_{n}(-x)$ on $\mathbb{R}^{3}$, that compactness of $\left\{\left|u_{n}\right|_{\mu_{1}}\right\}$ does not happen. Therefore subtracting subsequence, we have again by Lemma 1.1 of [13] that there exist $\lambda_{1}, \lambda_{2}>0$ and sequences $\left\{u_{n}^{1}\right\},\left\{u_{n}^{2}\right\} \subset H$ such that $\operatorname{dist}\left(\operatorname{Supp} u_{n}^{1}\right.$, Supp $\left.u_{n}^{2}\right) \longrightarrow \infty$, as $n \longrightarrow \infty$, and

$$
\begin{aligned}
\lim _{n \longrightarrow \infty}\left\|u_{n}-\left(u_{n}^{1}+u_{n}^{2}\right)\right\| & =0, \\
& \lim _{n \longrightarrow \infty}\left\|u_{n}-u_{n}^{1}\right\|=\lambda_{1} \quad \text { and } \quad \lim _{n \longrightarrow \infty}\left\|u_{n}-u_{n}^{2}\right\|=\lambda_{2} .
\end{aligned}
$$

Then it follows that

$$
\lim _{n \longrightarrow \infty} \nabla \Phi\left(u_{n}^{1}, V_{n}\right)=\lim _{n \longrightarrow \infty} \nabla \Phi\left(u_{n}^{2}, V_{n}\right)=0 .
$$

Therefore we have by (2) of Lemma 3 that

$$
\lim _{n \longrightarrow \infty} \Phi_{1}\left(u_{n}, V_{n}\right)=\lim _{n \longrightarrow \infty} \Phi_{1}\left(u_{n}^{1}, V_{n}\right)+\lim _{n \longrightarrow \infty} \Phi_{1}\left(u_{n}^{2}, V_{n}\right) \geq 2 c_{1} .
$$

Thus we find

$$
\lim _{n \longrightarrow \infty} \Phi_{1}\left(\mathcal{U}_{n}\right)=\lim _{n \longrightarrow \infty} \Phi_{1}\left(u_{n}, V_{n}\right)+\lim _{n \longrightarrow \infty} \Phi_{1}(U, V) \geq 3 c_{1} .
$$

This is a contradiction. Then case 1 does not happen. 
Case 2. Assume that $U \not \equiv 0$ and $\liminf _{n}\left\|u_{n}\right\|_{\mu_{1}}=0$. Then subtracting subsequences, we have $\lim _{n \longrightarrow \infty} \nabla_{v} \Phi\left(U_{n}, v_{n}\right)=\lim _{n \longrightarrow \infty} \nabla I_{2}\left(v_{n}\right)=0$. Assume that $\liminf \left\|_{n}\right\| v_{n} \|_{\mu_{2}}>0$. Then we have $\lim _{n} \longrightarrow \infty \Phi_{2}\left(u_{n}, v_{n}\right)=k c_{2}$ for some $k \in \mathbb{N}$. Then

$$
\lim _{n \longrightarrow \infty} \Phi\left(\mathcal{U}_{n}\right)=\Phi(U, V)+\lim _{n \longrightarrow \infty} \Phi_{2}\left(u_{n}, v_{n}\right)=\Phi(U, V)+k c_{2} \leq 2 c_{1}+c_{2}+\varepsilon .
$$

Then noting that $\Phi_{1}(U) \geq c_{1}$, we find by (1) that $\Phi(U, V) \in\left(c_{1}+l c_{2}-\delta_{2} / 2\right.$, $\left.c_{1}+l c_{2}+\delta_{2} / 2\right)$ for some $l \in \mathbb{N}$. Then

$$
2 c_{1}+c_{2}+\varepsilon=\lim _{n \longrightarrow \infty} \Phi\left(\mathcal{U}_{n}\right) \in\left(c_{1}+(l+k) c_{2}-\delta_{2} / 2, c_{1}+(l+k) c_{2}+\delta_{2} / 2\right) .
$$

Since $\varepsilon \in\left(0, \delta_{2} / 2\right)$, this contradicts to $(2.7)$. Therefore there exists a subsequence $\left\{v_{n_{i}}\right\} \subset\left\{v_{n}\right\}$ such that $\lim _{i \longrightarrow \infty}\left\|v_{n_{i}}\right\|=0$. That is $\lim _{i \longrightarrow \infty} \Phi\left(\mathcal{U}_{n_{i}}\right)=\Phi(U, V)$. This implies that $\left\{\mathcal{U}_{n_{i}}\right\}$ is a strongly convergent subsequence of $\left\{\mathcal{U}_{n}\right\}$.

Case 3. Assume that $U \equiv 0$. Then we have $\liminf _{n \rightarrow \infty}\left\|u_{n}\right\|>0$. Therefore $(2.9)$ holds. That is $\liminf _{n} \Phi\left(\mathcal{U}_{n}\right) \geq 2 c_{1}$. Then $\lim \sup _{n} \Phi_{2}\left(\mathcal{U}_{n}\right) \leq c_{2}+\varepsilon$. This implies $\liminf \operatorname{in}_{n}\left\|v_{n}\right\|=0$. In fact, if $\liminf _{n}\left\|v_{n}\right\|>0$, we have by the same argument as above that $\lim \sup _{n} \Phi_{2}\left(\mathcal{U}_{n}\right) \geq 2 c_{2}$. This is a contradiction. Therefore by the uniqueness of positive solution of $\left(P_{2}\right)$, we have

$$
\begin{aligned}
& \liminf _{n} \Phi_{2}\left(\mathcal{U}_{n}\right)=\liminf _{n} \Phi_{2}(0, V)=\liminf _{n} I_{2}(V)=c_{2} \quad \text { and } \\
& \liminf _{n} \Phi_{1}\left(\mathcal{U}_{n}\right)=2 \liminf _{n} I_{1}\left(u_{n}\right)=2 c_{1} .
\end{aligned}
$$

That is $\lim _{n \longrightarrow \infty} \Phi\left(\mathcal{U}_{n}\right)=2 c_{1}+c_{2}$. This is a contradiction. Therefore $U \equiv 0$ can not happen. This completes the proof.

Lemma 5. For given $\varepsilon>0$, there exists $\beta_{\varepsilon} \in\left(\beta_{1}, 0\right)$ such that for each $\beta \in\left(\beta_{\varepsilon}, 0\right)$ and for each $x \in \mathbb{R}^{3} \backslash\{0\}$,

$$
\Phi\left(\mathcal{N}\left(U_{1, x}+U_{1,-x}, U_{2}\right)\right)<2 c_{1}+c_{2}+\varepsilon
$$

and

$$
\Phi\left(\mathcal{N}\left(U_{1}, U_{2}\right)\right)<\frac{7}{4} c_{1}+c_{2}
$$

Proof. Let $\beta \in\left(\beta_{1}, 0\right)$. Let $x \in \mathbb{R}^{3} \backslash\{0\}$ and $t_{1}(x), t_{2}(x)>0$ such that $\left(t_{1}(x)\left(U_{1, x}+\right.\right.$ $\left.\left.U_{1,-x}\right), t_{2}(x) U_{2}\right) \in \mathcal{M}_{2}$. That is

$$
\left(\begin{array}{c}
t_{1}(x)^{2} \\
t_{2}(x)^{2}
\end{array}\right)=A_{x}^{-1}\left(\begin{array}{c}
\left\|U_{1, x}+U_{1,-x}\right\|_{\mu_{1}}^{2} \\
\left\|U_{2}\right\|_{\mu_{2}}^{2}
\end{array}\right)
$$

where

$$
A_{x}=\left(\begin{array}{cc}
\left|U_{1, x}+U_{1,-x}\right|_{4}^{4} & \beta \int_{\mathbb{R}^{3}}\left(U_{1, x}+U_{1,-x}\right)^{2} U_{2}^{2} \\
\beta \int_{\mathbb{R}^{3}}\left(U_{1, x}+U_{1,-x}\right)^{2} U_{2}^{2} & \left|U_{2}\right|_{4}^{4}
\end{array}\right) .
$$

Since $\beta \in\left(\beta_{1}, 0\right) \subset(-1,0)$, we can see by Schwartz's inequality that inf $\operatorname{in}_{x \in \mathbb{R}^{3}}\left|A_{x}\right|>$ 0 . Then one can see that there exists $M>0$ such that

$$
\max \left\{t_{1}(x), t_{2}(x)\right\}<M \text { for all } x \in \mathbb{R}^{3} \backslash\{0\},
$$


where $M$ is independent of $\beta \in\left(\beta_{1}, 0\right)$. Then we have

$$
L=\max _{x \in \mathbb{R}^{3}}\left\{t_{2}(x)^{2} \int_{\mathbb{R}^{3}}\left(U_{1, x}+U_{1,-x}\right)^{2} U_{2}^{2}, t_{1}(x)^{2} \int_{\mathbb{R}^{3}}\left(U_{1, x}+U_{1,-x}\right)^{2} U_{2}^{2}\right\}<\infty .
$$

Then by the definition of $U_{1}$, we have by

$$
\begin{aligned}
\left\|U_{1, x}+U_{1,-x}\right\|_{\mu_{1}}^{2}= & \left\|U_{1, x}\right\|_{\mu_{1}}^{2}+\left\|U_{1,-x}\right\|_{\mu_{1}}^{2} \\
& +2\left\langle U_{1, x}, U_{1,-x}\right\rangle_{\mu_{1}} \\
= & \left|U_{1, x}\right|_{4}^{4}+\left|U_{1,-x}\right|_{4}^{4}+2 \int_{R^{3}} U_{1, x}^{3} U_{1,-x} \\
= & \left|U_{1, x}\right|_{4}^{4}+\left|U_{1,-x}\right|_{4}^{4}+2 \int_{R^{3}} U_{1, x} U_{1,-x}^{3} .
\end{aligned}
$$

Then noting that $\int_{\mathbb{R}^{3}} U_{1, x}^{2} U_{1,-x}^{2} \leq\left|U_{1, x}\right|_{4}^{4}$, we find

$$
\frac{\left\|U_{1, x}+U_{1,-x}\right\|_{\mu_{1}}^{4}}{\left|U_{1, x}+U_{1,-x}\right|_{4}^{4}} \leq\left|U_{1, x}\right|_{4}^{4}+\left|U_{1,-x}\right|_{4}^{4}=8 c_{1} \quad \text { for } \quad x \in \mathbb{R}^{3} .
$$

Therefore we have by (2.12), (2.14) and the inequality above that

$$
\begin{aligned}
t_{1}(x)^{2}\left\|U_{1, x}+U_{1,-x}\right\|_{\mu_{1}}^{2} & \leq\left(\frac{\left\|U_{1, x}+U_{1,-x}\right\|_{\mu_{1}}^{2}-\beta L}{\left|U_{1, x}+U_{1,-x}\right|_{4}^{4}}\right)\left\|U_{1, x}+U_{1,-x}\right\|_{\mu_{1}}^{2} \\
& \leq 8 c_{1}\left(1-\frac{\beta L}{\left|U_{1}\right|_{4}^{4}}\right) .
\end{aligned}
$$

Similarly, we have that

$$
t_{2}(x)^{2}\left\|U_{2}\right\|_{\mu_{1}}^{2} \leq 4 c_{2}\left(1-\frac{\beta L}{\left|U_{2}\right|_{4}^{4}}\right) .
$$

Then combining the inequalities above, we have

$$
\Phi\left(\mathcal{N}\left(U_{1, x}+U_{1,-x}, U_{2}\right)\right) \leq 2 c_{1}+c_{2}-\left(\frac{\beta L c_{1}}{\left|U_{1}\right|_{4}^{4}}+\frac{\beta L c_{2}}{\left|U_{2}\right|_{4}^{4}}\right)=2 c_{1}+c_{2}-8 \beta L .
$$

Then for given $\varepsilon>0$, we can choose $\beta_{\varepsilon}<0$ with $\left|\beta_{2}\right|$ sufficiently small that $(2.10)$ holds. The inequality (2.11) can be proved by a parallel argument as above. Then we omit the proof.

\section{Proof of Theorem 1 for $i=2$.}

Throughout this section we assume that $\beta \in\left(\beta_{1}, 0\right)$. We put

$$
b_{R}(U)=\int_{\mathbb{R}^{3} \backslash B_{R}(0)}|U|_{\mu_{1}}^{2} \quad \text { for } \quad U \in H \quad \text { and } \quad R>0
$$


and

$$
\Lambda_{2, \varepsilon}(R)=\left\{\mathcal{U}=(U, V) \in \Phi^{2 c_{1}+c_{2}+\varepsilon} \cap \mathcal{M}_{2}: b_{R}(U) \geq 8 c_{1}-\min \left\{\frac{1}{2 m_{4}^{4}}, c_{1}\right\}\right\}
$$

for each $\varepsilon>0$ and $R>0$.

Proposition 1. For $\varepsilon>0$ sufficiently small, there exists $\left(R_{\varepsilon}, \delta_{\varepsilon}, \alpha_{\varepsilon}, \gamma_{\varepsilon}\right) \in\left(\mathbb{R}^{+}\right)^{4}$ such that $\lim _{\varepsilon \longrightarrow 0} \delta_{\varepsilon}=\lim _{\varepsilon \longrightarrow 0} \alpha_{\varepsilon}=\lim _{\varepsilon \longrightarrow 0} \gamma_{\varepsilon}=0$ and each $\mathcal{U}=(U, V) \in$ $\Lambda_{2, \varepsilon}\left(R_{\varepsilon}\right)$ has the form

$$
\mathcal{U}=\left(\alpha\left(U_{1, x}+U_{1,-x}\right)+u, \gamma U_{2}+v\right)
$$

where $\alpha \in\left(1-\alpha_{\varepsilon}, 1+\alpha_{\varepsilon}\right), \gamma \in\left(1-\gamma_{\varepsilon}, 1+\gamma_{\varepsilon}\right)$,

$$
\begin{aligned}
|x| & \geq R_{\varepsilon}, \quad x=\mathcal{B}\left(\left.U\right|_{B_{R_{0}}(x)}\right), \\
\widehat{U}(z) & <\frac{1}{2}|\widehat{U}|_{\infty} \quad \text { for } \quad z \in \mathbb{R}^{3} \backslash \bigcup_{i= \pm 1} B_{R_{0}}(i x), \\
\widehat{V}(z) & <\frac{1}{2}|\widehat{V}|_{\infty} \quad \text { for } \quad z \in \mathbb{R}^{3} \backslash B_{R_{0}}(0),
\end{aligned}
$$

and

$$
(u, v) \in\left\{U_{1, x}, U_{1,-x}\right\}^{\perp} \times\left\{U_{2}\right\}^{\perp} \quad \text { with } \quad\|u\|_{\mu_{1}}^{2}+\|v\|_{\mu_{2}}^{2} \leq \delta_{\varepsilon} .
$$

Proof. Let $r>1$ and $z \in \mathbb{R}^{3}$ with $|z|=1$, we put

$$
B_{r, z}=\left(B_{r+1}(0) \cup\left\{x \in \mathbb{R}^{3}:\left|\langle x, z\rangle_{\mathbb{R}^{3}}\right|<1\right\}\right) \backslash \overline{B_{r}(0)} .
$$

Let $\varphi_{r, z} \in C^{\infty}\left(\mathbb{R}^{3} ;[0,1]\right)$ such that $\varphi_{r, z}(x)=\varphi_{r, z}(-x)$ on $\mathbb{R}^{3},|\nabla \varphi|_{\infty} \leq 4$, and

$$
\varphi_{r, z}=\left\{\begin{array}{lll}
1 & \text { on } & \mathbb{R}^{3} \backslash B_{r, z} \\
0 & \text { on } & \partial B_{r+\frac{1}{2}}(0) \cup\left\{x \in \mathbb{R}^{3} ;\langle x, z\rangle=0,|x| \geq r+\frac{1}{2}\right\} .
\end{array}\right.
$$

Then we have that there exists $C_{1}>1$ such that

$$
\max \left\{\int_{B_{r, z}}\left|\varphi_{r, z} U\right|_{\mu_{1}}^{2}, \int_{B_{r, z}}\left|U^{+}\right|^{4}\right\} \leq C_{1} \int_{B_{r, z}}|U|_{\mu_{1}}^{2}
$$

for $(r, z) \in \mathbb{R}^{+} \times \partial B_{1}(0)$ and $U \in \Phi^{2 c_{1}+2 c_{2}} \cap \mathcal{M}_{2}$.

Now let $\varepsilon \in\left(0, \min \left\{1 / 8 m_{4}^{4}, c_{1}\right\}\right), R_{\varepsilon}$ be a positive number such that $R_{\varepsilon}>$ $r+\frac{1}{2}$, and $\mathcal{U}=(U, V) \in \Lambda_{2, \varepsilon}\left(R_{\varepsilon}\right)$. From the definition of $\varphi_{r, z}$, we have that $\varphi_{r, z} U$ has the form

$$
\varphi_{r, z} U=W_{0}+W_{+}+W_{-},
$$

where $\operatorname{supp} W_{0}$, supp $W_{+}, \operatorname{supp} W_{-}$are disjoint, $\operatorname{supp} W_{0} \subset B_{r+\frac{1}{2}}(0)$ and $W_{+}(x)=$ $W_{-}(-x)$ for $x \in \mathbb{R}^{3}$. Since $\|V\|_{\mu_{2}}^{2} \geq 4 c_{2}$ by (1) of Lemma 3 ,

$$
\|U\|_{\mu_{1}}^{2}=4 \Phi_{1}(\mathcal{U}) \leq 8 c_{1}+4 \varepsilon .
$$


We also have

$$
\begin{aligned}
\left(\int_{B_{r+\frac{1}{2}}(0)}\left|U^{+}\right|^{4}\right)^{\frac{1}{2}} & \leq m_{4}^{2} \int_{B_{r+\frac{1}{2}}(0)}|U|_{\mu_{1}}^{2} \leq m_{4}^{2}\left(8 c_{1}+4 \varepsilon-\int_{\mathbb{R}^{3} \backslash B_{r+\frac{1}{2}}(0)}|U|_{\mu_{1}}^{2}\right) \\
& \leq m_{4}^{2}\left(8 c_{1}+4 \varepsilon-b_{R_{\varepsilon}}(U)\right)<\frac{1}{m_{4}^{2}} .
\end{aligned}
$$

Then we find

$$
\begin{aligned}
\int_{B_{r+\frac{1}{2}}(0)}\left|U^{+}\right|^{4} & =\frac{1}{m_{4}^{2}}\left(m_{4}^{2}\left(\int_{B_{r+\frac{1}{2}}(0)}\left|U^{+}\right|^{4}\right)^{\frac{1}{2}}\right)\left(\int_{B_{r+\frac{1}{2}}(0)}\left|U^{+}\right|^{4}\right)^{\frac{1}{2}} \\
& <\int_{B_{r+\frac{1}{2}}(0)}|U|_{\mu_{1}}^{2} .
\end{aligned}
$$

Then

$$
\int_{B_{r+\frac{1}{2}}(0)}|U|_{\mu_{1}}^{2}>\int_{B_{r+\frac{1}{2}}(0)}\left|U^{+}\right|^{4}+\beta \int_{\mathbb{R}^{3}}\left(U^{+}\right)^{2}\left(V^{+}\right)^{2} .
$$

Therefore noting that $\mathcal{U} \in \mathcal{M}_{2}$, we have

$$
\int_{\mathbb{R}^{3} \backslash B_{r+\frac{1}{2}}(0)}\left(|U|_{\mu_{1}}^{2}-\left|U^{+}\right|^{4}\right)<0 .
$$

On the other hand, we have from (3.5) and (3.6) that

$$
\begin{aligned}
\left\|W_{+}\right\|_{\mu_{1}}^{2} & \leq \frac{1}{2}\left(\int_{\mathbb{R}^{3} \backslash B_{r+\frac{1}{2}}(0)}|U|_{\mu_{1}}^{2}+\int_{B_{r, z}}\left|\varphi_{r, z} U\right|_{\mu_{1}}^{2}\right) \\
& \leq \frac{1}{2}\left(\int_{\mathbb{R}^{3} \backslash B_{r+\frac{1}{2}}(0)}|U|_{\mu_{1}}^{2}+C_{1} \int_{B_{r, z}}|U|_{\mu_{1}}^{2}\right),
\end{aligned}
$$

and

$$
\begin{aligned}
\left|W_{+}^{+}\right|_{4}^{4} & \geq \frac{1}{2}\left(\int_{\mathbb{R}^{3} \backslash B_{r+\frac{1}{2}}(0)}\left|U^{+}\right|^{4}-\int_{B_{r, z}}\left|U^{+}\right|^{4}\right) \\
& \geq \frac{1}{2}\left(\int_{\mathbb{R}^{3} \backslash B_{r+\frac{1}{2}}(0)}\left|U^{+}\right|^{4}-C_{1} \int_{B_{r, z}}|U|_{\mu_{1}}^{2}\right) .
\end{aligned}
$$


Then noting $\beta<0$, we have from (3.8), (3.9) and (3.10) that

$$
\begin{aligned}
\left\|W_{+}\right\|_{\mu_{1}}^{2} & =\int_{\mathbb{R}^{3} \backslash B_{r+\frac{1}{2}}(0)}\left|W_{+}\right|_{\mu_{1}}^{2} \leq \frac{1}{2}\left(\int_{\mathbb{R}^{3} \backslash B_{r+\frac{1}{2}}(0)}|U|_{\mu_{1}}^{2}+C_{1} \int_{B_{r, z}}|U|_{\mu_{1}}^{2}\right) \\
& \leq \frac{1}{2}\left(\int_{\mathbb{R}^{3} \backslash B_{r+\frac{1}{2}}(0)}\left|U^{+}\right|^{4}+C_{1} \int_{B_{r, z}}|U|_{\mu_{1}}^{2}\right) \leq\left|W_{+}\right|_{4}^{4}+C_{1} \int_{B_{r, z}}|U|_{\mu_{1}}^{2} .
\end{aligned}
$$

Now let $t>0$ such that

$$
\left\|W_{+}\right\|^{2}=t^{2}\left|W_{+}^{+}\right|_{4}^{4}
$$

Then we have

$$
t^{2}=\frac{\left\|W_{+}\right\|^{2}}{\left|W_{+}^{+}\right|_{4}^{4}} \leq \frac{\left\|W_{+}\right\|^{2}}{\left\|W_{+}\right\|^{2}-C_{1} \int_{B_{r, z}}|U|_{\mu_{1}}^{2}} \leq 1+\frac{\alpha(r, z)}{\left\|W_{+}\right\|^{2}-\alpha(r, z)},
$$

where $\alpha(r, z)=C_{1} \int_{B_{r, z}}|U|_{\mu_{1}}^{2}$. Since (3.5), (3.7) and $t W_{+} \in S_{1}$ hold, we find

$$
\begin{aligned}
4 c_{1} \leq t^{2}\left\|W_{+}\right\|_{\mu_{1}}^{2} & \leq\left(1+\frac{\alpha(r, z)}{\left\|W_{+}\right\|^{2}-\alpha(r, z)}\right)\left(\frac{1}{2} \int_{\mathbb{R}^{3} \backslash B_{r, z}}|U|_{\mu_{1}}^{2}+\int_{B_{r, z}}\left|\varphi_{r, z}^{+} U\right|_{\mu_{1}}^{2}\right) \\
& \leq\left(1+\frac{\alpha(r, z)}{\left\|W_{+}\right\|^{2}-\alpha(r, z)}\right)\left(4 c_{1}+2 \varepsilon+\alpha(r, z)\right) .
\end{aligned}
$$

We now choose $R_{\varepsilon}>0$ sufficiently large that there exists $(r, z) \in\left(R_{\varepsilon} / 2, R_{\varepsilon}\right) \times$ $\partial B_{1}(0)$ such that

$$
\alpha(r, z)=C_{1} \int_{B_{r, z}}|U|_{\mu_{1}}^{2}<\varepsilon
$$

Let $(r, z)$ satisfy $(3.12)$. Then

$$
4 c_{1} \leq t^{2}\left\|W_{+}\right\|_{\mu_{1}}^{2} \leq\left(1+\frac{\varepsilon}{\left\|W_{+}\right\|^{2}-\varepsilon}\right)\left(4 c_{1}+3 \varepsilon\right) .
$$

Since

$$
\begin{aligned}
\left\|W_{+}\right\|_{\mu_{1}}^{2} & \geq \frac{1}{2} \int_{R^{N} \backslash B_{r, z}}|U|_{\mu_{1}}^{2} \\
& =\frac{1}{2}\left(\|U\|_{\mu_{1}}^{2}-\int_{B_{r, z}}|U|_{\mu_{1}}^{2}\right) \\
& \geq \frac{1}{2}\left(b_{R_{\varepsilon}}(U)-\frac{\alpha(r, z)}{C_{1}}\right) \\
& \geq \frac{1}{2}\left(7 c_{1}-\frac{\varepsilon}{C_{1}}\right) \geq 3 c_{1},
\end{aligned}
$$


we have by (3.13) that

$$
4 c_{1} \leq t^{2}\left\|W_{+}\right\|_{\mu_{1}}^{2} \leq\left(1+\frac{\varepsilon}{3 c_{1}-\varepsilon}\right)\left(4 c_{1}+3 \varepsilon\right) .
$$

Then by Lemma 1 that for $\varepsilon>0$ sufficiently small, $t W_{+}$has the form

$$
t W_{+}=\widetilde{\alpha} U_{1, \widetilde{x}}+\widetilde{u}
$$

where $\widetilde{\alpha} \longrightarrow 1$ and $\|\widetilde{u}\|_{\mu_{1}} \longrightarrow 0$ as $\varepsilon \longrightarrow 0$ and $\widetilde{x} \in \mathbb{R}^{3}$ satisfies $\mathcal{B}\left(W_{+}\right)=\widetilde{x}$ and $|\widetilde{x}| \longrightarrow \infty$, as $\varepsilon \longrightarrow 0$. On the other hand, we have by (3.7) and (3.9) that

$$
\left\|W_{+}\right\|_{\mu_{1}}^{2} \leq \frac{1}{2}\left(8 c_{1}+4 \varepsilon+\varepsilon\right) \leq 4 c_{1}+4 \varepsilon .
$$

Then

$$
\frac{c_{1}}{c_{1}+\varepsilon} \leq \frac{4 c_{1}}{\left\|W_{+}\right\|_{\mu_{1}}^{2}} \leq t^{2} .
$$

That is we have by (3.11) and the inequalities above that $t \rightarrow 1$ as $\varepsilon \rightarrow 0$. Since $\varphi_{r, z}=1$ on $\mathbb{R}^{3} \backslash B_{r, z}$ and $(3.12)$ holds, we find $\left\|U-\varphi_{r, z} U\right\|_{\mu_{1}}^{2} \longrightarrow 0$ as $\varepsilon \longrightarrow 0$. Then noting that $\left\|W_{+}+W_{-}\right\|^{2} \longrightarrow 8 c_{1}$ as $\varepsilon \longrightarrow 0$ by (3.14), and $\|U\|_{\mu_{1}}^{2} \leq 8 c_{1}+4 \varepsilon$, we find that $\left\|W_{0}\right\|_{\mu_{1}}^{2} \longrightarrow 0$, as $\varepsilon \longrightarrow 0$. Therefore $U$ has the form

$$
U=\widetilde{\alpha}\left(U_{1, \widetilde{x}}+U_{1,-\widetilde{x}}\right)+\widehat{u},
$$

where $\widehat{u} \in H$ with $\|\widehat{u}\|_{\mu_{1}}^{2} \longrightarrow 0$ and $\widetilde{\alpha} \longrightarrow 1$, as $\varepsilon \longrightarrow 0$. Then we have as in the proof of Lemma 1 that there exists $x \in \mathbb{R}^{3}, \alpha \in(0,2)$ and $u \in\left\{U_{1, x}, U_{1,-x}\right\}^{\perp}$ such that $x=\mathcal{B}\left(\left.U\right|_{B_{R_{0}}(x)}\right)$ with $|x| \geq R_{\varepsilon}$,

$$
U=\alpha\left(U_{1, x}+U_{1,-x}\right)+u,
$$

and it follows that $\|u\|_{\mu_{1}}^{2} \longrightarrow 0$ and $\alpha \longrightarrow 1$ as $\varepsilon \longrightarrow 0$. From (1.4), One can see that $\widehat{U}(z)<\frac{1}{2}|\widehat{U}|_{\infty}$ on $\mathbb{R}^{3} \backslash\left(B_{R_{0}}(x) \cup B_{R_{0}}(-x)\right)$. Then $(3.2)$ holds. By a parallel argument, one can see that the assertions for $V$ also follows, and then (3.3) and (3.4) holds.

Remark 4. By (3.2) and the definition of $\mathcal{B}$, one can see that for each $\mathcal{U} \in$ $\Lambda_{2, \varepsilon}\left(R_{\varepsilon}\right),(x,-x) \in \mathbb{R}^{3} \times \mathbb{R}^{3}$ in (3.1) is uniquely determined, and the mapping $\mathcal{U} \in \Lambda_{2, \varepsilon}\left(R_{\varepsilon}\right) \rightarrow(x,-x) \in \mathbb{R}^{3} \times \mathbb{R}^{3}$ is continuous. We define a continuous mapping $\eta: \Lambda_{2, \varepsilon}\left(R_{\varepsilon}\right) \longrightarrow \mathbb{R}^{+}$by

$$
\eta(\mathcal{U})=|x| \quad \text { for } \quad \mathcal{U} \in \Lambda_{2, \varepsilon}\left(R_{\varepsilon}\right) .
$$

We next prove the following Proposition.

Proposition 2. There exists $M_{0}>0$ satisfying that for $\varepsilon>0$ sufficiently small,

$$
\Phi(\mathcal{U}) \geq 2 c_{1}+c_{2}-\beta M_{0} e^{-2 \sqrt{\mu_{2}}|x|} \quad \text { for each } \mathcal{U} \in \Lambda_{2, \varepsilon}\left(R_{\varepsilon}\right),
$$

where $x \in \mathbb{R}^{3}$ such that $\mathcal{U}$ has the form (3.1).

To prove Proposition 2, we need following two lemmas. 
Lemma 6. There exists $k_{1}>0$ such that for $\varepsilon>0$ sufficiently small, each $\mathcal{U}=$ $(U, V) \in \mathcal{M}_{2} \cap \Phi^{2 c_{1}+c_{2}+\varepsilon}$ satisfies

$$
\Phi_{2}(\mathcal{U}) \geq c_{2}+k_{1}\|v\|_{\mu_{1}}^{2},
$$

where $v \in\left\{U_{2}\right\}^{\perp}$ such that $V=\alpha U_{2}+v$ for some $\alpha \in \mathbb{R}$.

Proof. Let $\varepsilon>0$ sufficiently small and $\mathcal{U}=(U, V) \in \mathcal{M}_{2} \cap \Phi^{2 c_{1}+c_{2}+\varepsilon}$. We have from Proposition 1 that $V$ has the form $V=(1+\theta) U_{2}+v$, where $v \in\left\{U_{2}\right\}^{\perp}$ and $|\theta|$ is small. If $\theta \geq 0$, then the assertion holds with $k_{1}=1$. We assume that $\theta<0$. Since $v(x)=v(-x)$ on $\mathbb{R}^{3}$ and $\frac{\partial U_{2}}{\partial x_{i}}$ is odd for each $1 \leq i \leq 3$, we have that $\left\langle v, \frac{\partial U_{2}}{\partial x_{i}}\right\rangle=0$ for $1 \leq i \leq 3$. Then noting that

$$
\left\langle-\Delta U_{2}+\mu_{2} U_{2}, v\right\rangle=\left\langle U_{2}^{3}, v\right\rangle=0,
$$

we have

$$
\begin{aligned}
\left\|(1+\theta) U_{2}+v\right\|_{\mu_{2}}^{2}= & \left.\int_{\mathbb{R}^{3}}\left[\left((1+\theta) U_{2}+v\right)^{+}\right)^{4}+\beta\left(U^{+}\right)^{2}\left(V^{+}\right)^{2}\right] \\
\leq & \int_{\mathbb{R}^{3}}\left[(1+\theta)^{4} U_{2}^{4}+6(1+\theta)^{2} U_{2}^{2} v^{2}+4(1+\theta) U_{2} v^{3}+v^{4}\right. \\
& \left.+\beta\left(U^{+}\right)^{2}\left(V^{+}\right)^{2}\right] .
\end{aligned}
$$

Then since $\left\|U_{2}\right\|_{\mu_{2}}^{2}=\int_{R^{3}} U_{2}^{4}$, we have

$\theta\left\|U_{2}\right\|_{\mu_{2}}^{2}+O\left(\theta^{2}\right) \geq \frac{1}{2}\left(\|v\|_{\mu_{2}}^{2}-\int_{\mathbb{R}^{3}}\left[6 U_{2}^{2} v^{2}+4(1+\theta) U_{2} v^{3}+v^{4}+\beta\left(U^{+}\right)^{2}\left(V^{+}\right)^{2}\right]\right.$.

Then by (2.2),

$$
\begin{aligned}
\left\|(1+\theta) U_{2}+v\right\|_{\mu_{2}}^{2}= & (1+\theta)^{2}\left\|U_{2}\right\|_{\mu_{2}}^{2}+\|v\|_{\mu_{2}}^{2} \\
\geq & 4 c_{2}+2\|v\|_{\mu_{2}}^{2}-\int_{R^{3}}\left[6 U_{2}^{2} v^{2}+4(1+\theta) U_{2} v^{3}+v^{4}\right. \\
& \left.+\beta\left(U^{+}\right)^{2}\left(V^{+}\right)^{2}\right] \\
\geq & 4 c_{2}+2\left(\|v\|_{\mu_{2}}^{2}-3 \int_{R^{3}} U_{2}^{2} v^{2}\right)+o\left(\|v\|_{\mu_{2}}^{2}\right) \\
& -\beta \int_{\mathbb{R}^{3}}\left(U^{+}\right)^{2}\left(V^{+}\right)^{2} \geq 4 c_{2}+2 \lambda\|v\|_{\mu_{2}}^{2}+o\left(\|v\|_{\mu_{2}}^{2}\right) .
\end{aligned}
$$

Since $\|v\|_{\mu_{2}}^{2} \rightarrow 0$, as $\varepsilon \rightarrow 0$, the assertion holds with $k_{1}=\lambda / 3$. This completes the proof.

Here we fix $\rho \in\left(0, \sqrt{\mu_{1}} / 2\right)$ such that

$$
\sqrt{\mu_{2}}<\sqrt{\mu_{1}}-2 \rho .
$$


Lemma 7. There exists $k_{2}>0$ such that for $\varepsilon>0$ sufficiently small,

$$
\begin{aligned}
\Phi_{1}(\mathcal{U}) & \geq 2 c_{1}-\frac{\beta}{8} \int_{\mathbb{R}^{3}}\left(U^{+}\right)^{2}\left(V^{+}\right)^{2}-k_{2} e^{-2\left(\sqrt{\mu_{1}}-\rho\right)|x|} \\
\mathcal{U} & =(U, V) \in \Lambda_{2, \varepsilon}\left(R_{\varepsilon}\right),
\end{aligned}
$$

where $x \in \mathbb{R}^{3}$ is the element satisfying (3.1).

Proof. For $r \geq 1$ and $x \in \partial B_{1}(0)$, we put

$$
\Omega_{0}(r, x)=\left\{z \in \mathbb{R}^{3}:|\langle x, z\rangle| \leq r\right\}, \quad \Omega_{ \pm}(r, x)=\left\{z \in \mathbb{R}^{3}:\langle \pm x, z\rangle \geq r\right\}
$$

and

$$
a_{n, x}(U)=\int_{\Omega_{0}(n, x)}|U|_{\mu_{1}}^{2} d x \quad \text { for each } n \geq 1 \quad \text { and } \quad U \in H .
$$

We also fix functions $\psi_{n, z}^{ \pm} \in C^{\infty}\left(\mathbb{R}^{3},[0,1]\right)$ such that

$$
\psi_{n, x}^{ \pm}(z)=1 \quad \text { on } \quad \Omega_{ \pm}(n+1, x) \quad \text { and } \quad \psi_{n, x}^{ \pm}(z)=0 \quad \text { on } \quad \mathbb{R}^{3} \backslash \Omega_{ \pm}(n, x)
$$

and $\left|\nabla \psi_{n, x}^{ \pm}\right| \leq 2$ on $\mathbb{R}^{3}$.

Step 1: We first show that there exists $C_{1}>0$ such that for $\varepsilon>0$ sufficiently small and $\mathcal{U}=(U, V) \in \Lambda_{2, \varepsilon}\left(R_{\varepsilon}\right)$, the following inequality holds:

$$
\begin{aligned}
\Phi_{1}(U)= & \frac{1}{4}\left\|U_{1, x}+U_{1,-x}+u\right\|_{\mu_{1}}^{2} \\
\geq & \frac{1}{4}\left(\int_{\Omega_{+}(n, x)}\left|U_{1, x}+\psi_{n, x}^{+}\left(U_{1,-x}+u\right)\right|_{\mu_{1}}^{2}\right. \\
& +\int_{\Omega_{-}(n, x)}\left|U_{1,-x}+\psi_{n, x}^{-}\left(U_{1, x}+u\right)\right|_{\mu_{1}}^{2} \\
& \left.+\int_{\Omega_{0}(n, x)}|U|_{\mu_{1}}^{2}-2 C_{1}\left(a_{n+1, x}(U)-a_{n, x}(U)\right)-2 C_{1} e^{-2 \sqrt{\mu_{1}}(|x|-n)}\right)
\end{aligned}
$$

for each $1 \leq n \leq\left(\rho / \sqrt{\mu_{1}}\right)|x|$, where $x \in \mathbb{R}^{3}$ satisfying (3.1) and $u \in H$ such that $U=U_{1, x}+U_{1,-x}+u$. Now let $\varepsilon>0, \mathcal{U}=(U, V) \in \Lambda_{2, \varepsilon}\left(R_{\varepsilon}\right)$ and $1 \leq n \leq$ $\left(\rho / \sqrt{\mu_{1}}\right)|x|$. Noting that $\left|\nabla \psi_{n, x}^{ \pm}\right| \leq 2$, we have

$$
\begin{aligned}
\int_{\Omega_{0}(n+1, x) \backslash \Omega_{0}(n, x)} & \left|\psi_{n, x}^{+} U\right|_{\mu_{1}}^{2} \\
& \leq 2 \int_{\Omega_{0}(n+1, x) \backslash \Omega_{0}(n, x)}\left(|U|_{\mu_{1}}^{2}+4|U|^{2}\right) \quad \text { for } \quad U \in H .
\end{aligned}
$$

By choosing $\varepsilon$ sufficiently small, we have by Proposition 1 that $U$ has the form $U=U_{1, x}+U_{1,-x}+u$. We may assume that $|x|$ is sufficiently large and $\|u\|_{\mu_{1}}<\frac{1}{4 m_{4}^{4}}$. Then by (1.3), we have that

$$
\left|U_{1, \pm x}(z)\right| \leq e^{-\sqrt{\mu_{1}}(|x|-n)} \quad \text { for } \quad z \in \Omega_{0}(n, x) \cup \Omega_{\mp}(n, x) .
$$


Since

$$
\begin{aligned}
\left|U_{1, \pm x}+\psi_{n, x}^{ \pm}\left(U_{1, \mp x}+u\right)\right|_{\mu_{1}}^{2} \leq & 2\left|U_{1, \pm x}-\psi_{n, x}^{ \pm} U_{1, \pm x}\right|_{\mu_{1}}^{2} \\
& +2\left|\psi_{n, x}^{ \pm}\left(U_{1, x}+U_{1,-x}+u\right)\right|_{\mu_{1}}^{2} \\
= & 2\left|U_{1, \pm x}-\psi_{n, x}^{ \pm} U_{1, \pm x}\right|_{\mu_{1}}^{2}+2\left|\psi_{n, x}^{ \pm} U\right|_{\mu_{1}}^{2}
\end{aligned}
$$

we have by (3.19) and (3.20) that there exists $C_{1}>0$ such that

$$
\begin{aligned}
\int_{\Omega_{0}(n+1, x) \backslash \Omega_{0}(n, x)} & \left(\left|U_{1, \pm x}+\psi_{n, x}^{ \pm}\left(U_{1, \mp}+u\right)\right|_{\mu_{1}}^{2}\right) \\
\leq & C_{1}\left(\int_{\Omega_{0}(n+1, x) \backslash \Omega_{0}(n, x)}|U|_{\mu_{1}}^{2} d x+e^{-2 \sqrt{\mu_{1}}(|x|-n)}\right) \\
= & C_{1}\left(\left(a_{n+1, x}(U)-a_{n, x}(U)\right)\right. \\
& \left.+e^{-2 \sqrt{\mu_{1}}(|x|-n)}\right) .
\end{aligned}
$$

Then

$$
\begin{aligned}
\int_{\Omega_{ \pm}(n, x)}|U|_{\mu_{1}}^{2} \geq & \int_{\Omega_{ \pm}(n+1, x)}|U|_{\mu_{1}}^{2} \\
= & \int_{\Omega_{ \pm}(n+1, x)}\left|U_{1, \pm x}+\psi_{n, x}^{ \pm}\left(U_{1, \mp x}+u\right)\right|_{\mu_{1}}^{2} \\
\geq & \int_{\Omega_{ \pm}(n, x)}\left|U_{1, \pm x}+\psi_{n, x}^{ \pm}\left(U_{1, \mp x}+u\right)\right|_{\mu_{1}}^{2} \\
& -\int_{\Omega_{0}(n+1, x) \backslash \Omega_{0}(n, x)}\left|U_{1, x}+\psi_{n, x}^{ \pm}\left(U_{1, \mp x}+u\right)\right|_{\mu_{1}}^{2} \\
\geq & \int_{\Omega_{ \pm}(n, x)}\left|U_{1, x}+\psi_{n, x}^{ \pm}\left(U_{1, \mp x}+u\right)\right|_{\mu_{1}}^{2} \\
& -C_{1}\left(\left(a_{n+1, x}(U)-a_{n, x}(U)\right)+e^{-2 \sqrt{\mu_{1}}(|x|-n)}\right) .
\end{aligned}
$$

Then noting that

$$
\Phi_{1}(\mathcal{U})=\frac{1}{4}\left(\int_{\Omega_{+}(n, x)}|U|_{\mu_{1}}^{2}+\int_{\Omega_{-}(n, x)}|U|_{\mu_{1}}^{2}+\int_{\Omega_{0}(n, x)}|U|_{\mu_{1}}^{2}\right),
$$

we find from (3.22) that (3.18) holds. Then to prove the assertion of Lemma 7, it is sufficient to show that the right hand side of inequality (3.18) is greater than that of (3.17).

Step 2: We show that inequality (3.17) holds in case that

$$
a_{n+1, x}(U)-a_{n, x}(U)<\left(8 C_{1}\right)^{-1} a_{n, x}(U)
$$


for some $1 \leq n \leq\left(\rho / \sqrt{\mu_{1}}\right)|x|$. That is we assume that there exists $n \in \mathbb{N}$ such that $1 \leq n \leq\left(\rho / \sqrt{\mu_{1}}\right)|x|$ and

$$
\int_{\Omega_{0}(n, x)}|U|_{\mu_{1}}^{2} \geq 8 C_{1}\left(a_{n+1, x}(U)-a_{n, x}(U)\right) .
$$

Recalling that $\int_{\Omega_{0}(n+1, x)}|u|_{\mu_{1}}^{2}<\frac{1}{4 m_{4}^{4}}$ and $\left|U_{1, \pm x}(z)\right|=O\left(e^{-\sqrt{\mu_{1}}(|x|-n)}\right)$ on $\Omega_{0}(n+$ $1, x)$, we have that for $|x|$ sufficiently large,

$$
\int_{\Omega_{0}(n+1, x)}|U|_{\mu_{1}}^{2}<\frac{1}{2 m_{4}^{4}} .
$$

Then noting that

$$
|U|_{L^{4}\left(\Omega_{0}(n+1, x)\right)}^{4} \leq m_{4}^{4}\left(\int_{\Omega_{0}(n+1, x)}|U|_{\mu_{1}}^{2}\right)^{2} \leq(1 / 2) \int_{\Omega_{0}(n+1, x)}|U|_{\mu_{1}}^{2},
$$

we find

$$
\int_{\Omega_{0}(n+1, x)}\left(|U|_{\mu_{1}}^{2}-U^{4}\right) \geq \frac{1}{2} \int_{\Omega_{0}(n+1, x)}|U|_{\mu_{1}}^{2} d x .
$$

Since $\mathcal{U} \in \mathcal{M}_{2}$, this yields

$$
\begin{aligned}
& \int_{\Omega_{+}(n+1, x)}|U|_{\mu_{1}}^{2} \\
& \quad \leq \int_{\Omega_{+}(n+1, x)}\left(U^{+}\right)^{4}+\frac{\beta}{2} \int_{\mathbb{R}^{3}}\left(U^{+}\right)^{2}\left(V^{+}\right)^{2}-\frac{1}{4} \int_{\Omega_{0}(n+1, x)}|U|_{\mu_{1}}^{2} d x .
\end{aligned}
$$

By (3.24),

$$
\begin{aligned}
\int_{\Omega_{+}(n, x)} \mid & U_{1, x}+\left.\psi_{n, x}^{+}\left(U_{1,-x}+u\right)\right|_{\mu_{1}} ^{2} \\
= & \int_{\Omega_{+}(n+1, x)}\left|U_{1, x}\right|_{\mu_{1}}^{2}+\int_{\Omega_{+}(n, x) \backslash \Omega_{+}(n+1, x)}\left|U_{1, x}+\psi_{n, x}^{+}\left(U_{1,-x}+u\right)\right|_{\mu_{1}}^{2} \\
\leq & \int_{\Omega_{+}(n+1, x)}\left(U^{+}\right)^{4}+\frac{\beta}{2} \int_{\mathbb{R}^{3}}\left(U^{+}\right)^{2}\left(V^{+}\right)^{2}-\frac{1}{4} \int_{\Omega_{0}(n+1, x)}|U|_{\mu_{1}}^{2} d x \\
& +\int_{\Omega_{+}(n, x) \backslash \Omega_{+}(n+1, x)}\left|U_{1, x}+\psi_{n, x}^{+}\left(U_{1,-x}+u\right)\right|_{\mu_{1}}^{2} \\
\leq & \int_{\Omega_{+}(n, x)}\left(\left(U_{1, x}+\psi_{n, x}^{+}\left(U_{1,-x}+u\right)\right)^{+}\right)^{4}+\frac{\beta}{2} \int_{\mathbb{R}^{3}}\left(U^{+}\right)^{2}\left(V^{+}\right)^{2} \\
& -\frac{1}{4} \int_{\Omega_{0}(n, x)}|U|_{\mu_{1}}^{2} d x+2 C_{1}\left(a_{n+1, x}(U)-a_{n, x}(U)\right)+e^{-2 \sqrt{\mu_{1}}(|x|-n)} .
\end{aligned}
$$


On the other hand, it follows from (3.21) that

$$
\begin{aligned}
& \left\|U_{1, \pm x}+\psi_{n, x}^{ \pm}\left(U_{1, \mp x}+u\right)\right\|_{\mu_{1}}^{2} \\
& \quad=\int_{\Omega_{ \pm}(n, x)}\left|U_{1, \pm x}+\psi_{n, x}^{ \pm}\left(U_{1, \mp x}+u\right)\right|_{\mu_{1}}^{2}+\int_{\mathbb{R}^{3} \backslash \Omega_{ \pm}(n, x)}|U|_{\mu_{1}}^{2} \\
& \quad \leq \int_{\Omega_{ \pm}(n, x)}\left|U_{1, \pm x}+\psi_{n, x}^{ \pm}\left(U_{1, \mp x}+u\right)\right|_{\mu_{1}}^{2}+O\left(e^{-2 \sqrt{\mu_{1}}(|x|-n)}\right) .
\end{aligned}
$$

Then we find

$$
\begin{aligned}
\left\|U_{1, x}+\psi_{n, x}^{+}\left(U_{1,-x}+u\right)\right\|_{\mu_{1}}^{2} \leq & \int_{\Omega_{+}(n, x)}\left(\left(U_{1, x}+\psi_{n, x}^{+}\left(U_{1,-x}+u\right)\right)^{+}\right)^{4} \quad(3.27) \\
& +\frac{\beta}{2} \int_{\mathbb{R}^{3}}\left(U^{+}\right)^{2}\left(V^{+}\right)^{2} \\
& -\frac{1}{4} \int_{\Omega_{0}(n, x)}|U|_{\mu_{1}}^{2} d x+2 C_{1}\left(a_{n+1, x}(U)-a_{n, x}(U)\right) \\
& +O\left(e^{-2 \sqrt{\mu_{1}}(|x|-n)}\right) .
\end{aligned}
$$

Now let $t>0$ such that $W=t^{-1}\left(U_{1, x}+\psi_{n, x}^{+}\left(U_{1,-x}+u\right)\right) \in S_{1}$. That is

$$
t^{-2} \int_{\mathbb{R}^{3}}\left|U_{1, x}+\psi_{n, x}^{+}\left(U_{1,-x}+u\right)\right|_{\mu_{1}}^{2}=\int_{\mathbb{R}^{3}} t^{-4}\left(\left(U_{1, x}+\psi_{n, x}^{+}\left(U_{1,-x}+u\right)\right)^{+}\right)^{4} .
$$

Since $|x|$ is large and $\|u\|_{\mu_{1}}^{2}$ is small, $t$ is close to 1 . Then we may assume that $t^{2} \leq 2$. Since $W \in S_{1}$, we have

$$
t^{-2} \int_{\mathbb{R}^{3}}\left|U_{1, x}+\psi_{n, x}^{+}\left(U_{1,-x}+u\right)\right|_{\mu_{1}}^{2}=\|W\|_{\mu_{1}}^{2} \geq 4 c_{1} .
$$

Then we find by (3.23), and (3.27) that there exists $C_{2}>0$ such that

$$
\begin{aligned}
\left\|U_{1, x}+\psi_{n, x}^{+}\left(U_{1,-x}+u\right)\right\|_{\mu_{1}}^{2}= & t^{2}\|W\|_{\mu_{1}}^{2} \leq t^{4} \int_{\mathbb{R}^{3}}\left(W^{+}\right)^{4} \\
& +\frac{\beta}{2} \int_{\mathbb{R}^{3}}\left(U^{+}\right)^{2}\left(V^{+}\right)^{2}+C_{2} e^{-2 \sqrt{\mu_{1}}(|x|-n)} .
\end{aligned}
$$

Thus it follows that

$$
\begin{aligned}
t^{2} & \geq \frac{\|W\|_{\mu_{1}}^{2}-\frac{\beta}{2 t^{2}} \int_{\mathbb{R}^{3}}\left(U^{+}\right)^{2}\left(V^{+}\right)^{2}-C_{2} e^{-2 \sqrt{\mu_{1}}(|x|-n)}}{\int_{R^{3}}\left(W^{+}\right)^{4}} \\
& \geq 1-\frac{\frac{\beta}{4} \int_{\mathbb{R}^{3}}\left(U^{+}\right)^{2}\left(V^{+}\right)^{2}+C_{2} e^{-2 \sqrt{\mu_{1}}(|x|-n)}}{\|W\|_{\mu_{1}}^{2}} .
\end{aligned}
$$


Then

$$
\begin{aligned}
\left\|U_{1, x}+\psi_{n, x}^{+}\left(U_{1,-x}+u\right)\right\|_{\mu_{1}}^{2} & =t^{2}\|W\|_{\mu_{1}}^{2} \\
& \geq\|W\|_{\mu_{1}}^{2}-\frac{\beta}{4} \int_{\mathbb{R}^{3}}\left(U^{+}\right)^{2}\left(V^{+}\right)^{2}-C_{2} e^{-2 \sqrt{\mu_{1}}(|x|-n)} \\
& \geq 4 c_{1}-\frac{\beta}{4} \int_{\mathbb{R}^{3}}\left(U^{+}\right)^{2}\left(V^{+}\right)^{2}-C_{2} e^{-2 \sqrt{\mu_{1}}(|x|-n)} .
\end{aligned}
$$

Similarly, we have

$$
\left\|U_{1,-x}+\psi_{n, x}^{-}\left(U_{1, x}+u\right)\right\|_{\mu_{1}}^{2} \geq 4 c_{1}-\frac{\beta}{4} \int_{\mathbb{R}^{3}}\left(U^{+}\right)^{2}\left(V^{+}\right)^{2}-C_{2} e^{-2 \sqrt{\mu_{1}}(|x|-n)} .
$$

Then combining (3.23), (3.26), (3.29) and (3.30) with (3.18), we obtain that (3.17) holds.

Step 3: Lastly, we show that inequality (3.17) holds in case that

$$
a_{n+1, x}(U)-a_{n, x}(U) \geq\left(8 C_{1}\right)^{-1} a_{n, x}(U) \text { for all } 1 \leq n \leq\left(\rho / \sqrt{\mu_{1}}\right)|x| .
$$

Assume that $a_{n+1, x}(U)-a_{n, x}(U) \geq\left(8 C_{1}\right)^{-1} a_{n, x}(U)$ for all $1 \leq n \leq\left(\rho / \sqrt{\mu_{1}}\right)|x|$. Then

$$
a_{n, x}(U) \geq d^{n-2} M \quad \text { for } \quad 1 \leq n \leq\left(\rho / \sqrt{\mu_{1}}\right)|x| .
$$

where $M=a_{2}(U)$ and $d=\left(1+\left(8 C_{1}\right)^{-1}\right)$. Then instead of $(3.27)$, we have that there exists $C_{3}>0$ such that

$$
\begin{aligned}
\left\|U_{1, x}+\psi_{1, x}^{+}\left(U_{1,-x}+u\right)\right\|_{\mu_{1}}^{2} \leq & \int_{\Omega_{+}(1, x)}\left(\left(U_{1, x}+\psi_{n, x}^{+}\left(U_{1,-x}+u\right)\right)^{+}\right)^{4} \\
& +\frac{\beta}{2} \int_{\mathbb{R}^{3}}\left(U^{+}\right)^{2}\left(V^{+}\right)^{2}+2 C_{1} M+C_{3} e^{-2 \sqrt{\mu_{1}}|x|},
\end{aligned}
$$

Let $W$ be as in the argument above. Then inequality (3.28) is rewritten as

$$
\begin{aligned}
t^{2} & \geq \frac{\|W\|_{\mu_{1}}^{2}-\frac{\beta}{4} \int_{\mathbb{R}^{3}}\left(V^{+}\right)^{2}\left(W^{+}\right)^{2}-2 C_{1} M-C_{3} e^{-2 \sqrt{\mu_{1}}|x|}}{\int_{R^{3}} W^{4}} \\
& =1-\frac{\frac{\beta}{4} \int_{\mathbb{R}^{3}}\left(V^{+}\right)^{2}\left(W^{+}\right)^{2}+2 C_{1} M+C_{3} e^{-2 \sqrt{\mu_{1}}|x|}}{\|W\|_{\mu_{1}}^{2}} .
\end{aligned}
$$

Since $|x|$ is large and $\|u\|_{\mu_{1}}$ is small by the assumption, we have $t$ is close to 1 and $W$ has the form

$$
W=\frac{1}{t}\left(U_{1, x}+\psi_{1, x}^{+}\left(U_{1,-x}+u\right)\right)=\alpha U_{1, x}+\widetilde{u} \quad \text { with } \quad \widetilde{u} \in\left\{U_{1, x}\right\}^{\perp} .
$$

Then noting that $|t-1|$ and $\left\|\psi_{1, x}^{+}\left(U_{1,-x}+u\right)\right\|_{\mu_{1}}^{2}$ are small, we have that $|\alpha-1|$ is small. Then we may assume that $\|\widetilde{u}\|_{\mu_{1}}^{2} \geq \frac{1}{4}\left\|\psi_{1, x}^{+}\left(U_{1,-x}+u\right)\right\|_{\mu_{1}}^{2}$. Let $n \in \mathbb{N}$ be the largest integer less than or equal to $\left(\rho / \sqrt{\mu_{1}}\right)|x|$. Recalling that $\left|U_{1,-x}(z)\right|=$ 
$O\left(e^{-\sqrt{\mu_{1}}(|x|-n)}\right)$ on $\Omega_{0}(n, x)$, we have that there exists $C_{4}>0$ such that

$$
\begin{aligned}
\left\|\psi_{1, x}^{+}\left(U_{1,-x}+u\right)\right\|_{\mu_{1}}^{2} & \geq \frac{1}{2} \int_{\Omega_{0}(n, x) \backslash \Omega_{0}(2, x)}|U|_{\mu_{1}}^{2}-\int_{\Omega_{0}(n, x) \backslash \Omega_{0}(2, x)}\left|U_{1, x}\right|^{2} \\
& \geq \frac{1}{2} \int_{\Omega_{0}(n, x) \backslash \Omega_{0}(2, x)}|U|_{\mu_{1}}^{2}-C_{4} e^{-2 \sqrt{\mu_{1}(|x|-n)}} \\
& \geq \frac{1}{2}\left(d^{n-2}-1\right) M-C_{4} e^{-2 \sqrt{\mu_{1}(|x|-n)}} .
\end{aligned}
$$

Therefore by Lemma 2, we have that for $|x|$ sufficiently large,

$$
\|W\|_{\mu_{1}}^{2} \geq 4 c_{1}+\frac{2}{3}\|\widetilde{u}\|_{\mu_{1}}^{2} \geq 4 c_{1}+\frac{1}{6}\left(d^{n-2}-1\right) M-C_{4} e^{\left.-2 \sqrt{\mu_{1}(}|x|-n\right)} .
$$

Then

$$
\begin{aligned}
& \left\|U_{1, x}+\psi_{1, x}^{+}\left(U_{1,-x}+u\right)\right\|_{\mu_{1}}^{2}=t^{2}\|W\|_{\mu_{1}}^{2} \\
\geq & \left(1-\frac{\frac{\beta}{4} \int_{\mathbb{R}^{3}}\left(U^{+}\right)^{2}\left(V^{+}\right)^{2}+2 C_{1} M}{\|W\|_{\mu_{1}}^{2}}\right)\|W\|_{\mu_{1}}^{2}-C_{3} e^{-2 \sqrt{\mu_{1}}|x|} \\
\geq & \left(1-\frac{2 C_{1} M}{\|W\|_{\mu_{1}}^{2}}\right)\|W\|_{\mu_{1}}^{2}-\frac{\beta}{4} \int_{\mathbb{R}^{3}}\left(U^{+}\right)^{2}\left(V^{+}\right)^{2}-C_{3} e^{-2 \sqrt{\mu_{1}}|x|} \\
\geq & \left(4 c_{1}+\frac{1}{12}\left(d^{n-2}-1\right) M\right)-2 C_{1} M-\frac{\beta}{4} \int_{\mathbb{R}^{3}}\left(U^{+}\right)^{2}\left(V^{+}\right)^{2} \\
& -C_{4} e^{\left.-2 \sqrt{\mu_{1}(}|x|-n\right)}-C_{3} e^{-2 \sqrt{\mu_{1}}|x|} \\
\geq & 4 c_{1}+\frac{1}{12}\left(d^{n-2}-24 C_{1}-1\right) M-\frac{\beta}{4} \int_{\mathbb{R}^{3}}\left(U^{+}\right)^{2}\left(V^{+}\right)^{2} \\
& -C_{4} e^{\left.-2 \sqrt{\mu_{1}(}|x|-n\right)}-C_{3} e^{-2 \sqrt{\mu_{1}}|x|} .
\end{aligned}
$$

Similarly, we have

$$
\begin{aligned}
\left\|U_{1,-x}+\psi_{n, x}^{-}\left(U_{1, x}+u\right)\right\|_{\mu_{1}}^{2} \geq & 4 c_{1}+\frac{1}{6}\left(d^{n-2}-12 C_{1}-1\right) M \\
& -\frac{\beta}{4} \int_{\mathbb{R}^{3}}\left(V^{+}\right)^{2}\left(W^{+}\right)^{2} \\
& -C_{4} e^{\left.-2 \sqrt{\mu_{1}}|x|-n\right)}-C_{3} e^{-2 \sqrt{\mu_{1}}|x|} .
\end{aligned}
$$

By choosing $\varepsilon>0$ sufficiently small, we have that $|x|$ is so large that we can choose $n \geq 1$ such that $d^{n-2}-12 C_{1}-1>0$. Then again we have (3.29). We also have (3.30). Then again by the same argument in Step 2, we have that inequality (3.17) holds. This completes the proof.

Proof of Proposition 2. Let $\varepsilon>0$ and $\mathcal{U}=(U, V) \in \Lambda_{2, \varepsilon}\left(R_{\varepsilon}\right)$. By Proposition 1, we may assume, by choosing $\varepsilon>0$ sufficiently small, that $\mathcal{U}$ has the form $\mathcal{U}=$ 
$\left(\alpha\left(U_{1, x}+U_{1-x}\right)+u, \gamma U_{2}+v\right)$, where $|x|$ is sufficiently large, $\alpha$ is close to 1 and $\|(u, v)\|^{2}<\delta_{\varepsilon}$. Then by Lemma 6 and Lemma 7 , we have

$$
\Phi(\mathcal{U}) \geq 2 c_{1}+c_{2}+k_{1}\|v\|^{2}-\frac{\beta}{8} \int_{\mathbb{R}^{3}}\left(U^{+}\right)^{2}\left(V^{+}\right)^{2}-k_{2} e^{-2\left(\sqrt{\mu_{1}}-\rho\right)|x|} .
$$

If $\|v\|_{\mu_{2}}^{2} \geq\left(2 k_{2} / k_{1}\right) e^{-2\left(\sqrt{\mu_{1}}-\rho\right)|x|}$, then we have that for $|x|$ sufficiently large,

$$
\Phi(\mathcal{U}) \geq 2 c_{1}+c_{2}+k_{2} e^{-\left(2 \sqrt{\mu_{1}}-\delta\right)|x|} .
$$

Now assume that $\|v\|_{\mu_{2}}^{2}<\left(2 k_{2} / k_{1}\right) e^{-2\left(\sqrt{\mu_{1}}-\rho\right)|x|}$. Then we have

$$
\begin{aligned}
\Phi(\mathcal{U}) & \left.\geq 2 c_{1}+c_{2}+k_{1}\|v\|_{\mu_{2}}^{2}-\frac{\beta}{8} \int_{\mathbb{R}^{3}} U^{2}\left(\gamma U_{2}+v\right)^{2}-k_{2} e^{-2\left(\sqrt{\mu_{1}}-\rho\right)|x|}\right) \\
& \geq 2 c_{1}+c_{2}+k_{1}\|v\|_{\mu_{2}}^{2}-\frac{\beta}{8} \int_{\mathbb{R}^{3}} U^{2}\left(\frac{\gamma^{2}}{2} U_{2}^{2}-v^{2}\right)-k_{2} e^{-2\left(\sqrt{\mu_{1}}-\rho\right)|x|} \\
& \geq 2 c_{1}+c_{2}-\frac{\beta \gamma^{2}}{8} \int_{\mathbb{R}^{3}} U^{2} U_{2}^{2}-k_{2} e^{-2\left(\sqrt{\mu_{1}}-\rho\right)|x|} .
\end{aligned}
$$

Recalling that $\sqrt{\mu_{2}}<\sqrt{\mu_{1}}-2 \rho$, we have by (1.3) that there exists $C_{1}>0$ such that

$$
\int_{\mathbb{R}^{3}}\left(U_{1, x}+U_{1,-x}\right)^{2} U_{2}^{2} \geq C_{1} e^{-2 \sqrt{\mu_{2}}|x|} .
$$

Similarly, we have that there exists $C_{2}>0$ such that

$$
\begin{aligned}
\left|\int_{\mathbb{R}^{3}}\left(U_{1, x}+U_{1,-x}\right) U_{2}^{2} u\right| & \leq\left(\int_{\mathbb{R}^{3}}\left(U_{1, x}+U_{1,-x}\right)^{2} U_{2}^{4}\right)^{\frac{1}{2}}\left(\int_{\mathbb{R}^{3}} u^{2}\right)^{\frac{1}{2}} \\
& \leq C_{2} e^{-\min \left\{\sqrt{\mu_{1}}, 2 \sqrt{\mu_{2}}\right\}|x|}\|u\|_{\mu_{1}} .
\end{aligned}
$$

Then noting that $U^{2} U_{2}^{2}=\left(U_{1, x}+U_{1,-x}\right)^{2} U_{2}^{2}+2\left(U_{1, x}+U_{1,-x}\right) U_{2}^{2} u+u^{2}$, we find

$$
\begin{aligned}
\Phi(\mathcal{U}) \geq & 2 c_{1}+c_{2}-\frac{\beta \gamma^{2}}{8}\left(C_{1} e^{-2 \sqrt{\mu_{2}}|x|}-C_{2} e^{-\min \left\{\sqrt{\mu_{1}}, 2 \sqrt{\mu_{2}}\right\}|x|}\|u\|_{\mu_{1}}+\|u\|_{\mu_{1}}^{2}\right) \\
& -k_{2} e^{-2\left(\sqrt{\mu_{1}}-\rho\right)|x|} .
\end{aligned}
$$

If $\min \left\{\sqrt{\mu_{1}}, 2 \sqrt{\mu_{2}}\right\}=2 \sqrt{\mu_{2}}$, then noting that $\|u\|_{\mu_{1}}^{2} \longrightarrow 0$ and $\gamma \longrightarrow 1$ as $\varepsilon \longrightarrow 0$, we have

$$
\Phi(\mathcal{U}) \geq 2 c_{1}+c_{2}-\frac{\beta C_{2}}{16} e^{-2 \sqrt{\mu_{2}}|x|}
$$

for $\varepsilon>0$ sufficiently small. Suppose that $\min \left\{\sqrt{\mu_{1}}, 2 \sqrt{\mu_{2}}\right\}=\sqrt{\mu_{1}}$. Then in case that $\|u\|_{\mu_{1}} \geq e^{-\sqrt{\mu_{2}}|x|}$, we have

$$
\begin{aligned}
\Phi(\mathcal{U}) \geq & 2 c_{1}+c_{2}-\frac{\beta \gamma^{2}}{8}\left(C_{1} e^{-2 \sqrt{\mu_{2}}|x|}+e^{-\sqrt{\mu_{2}}|x|}\left(e^{-\sqrt{\mu_{2}}|x|}-C_{2} e^{-\sqrt{\mu_{1}}|x|}\right)\right) \\
& -k_{2} e^{-2\left(\sqrt{\mu_{1}}-\rho\right)|x|}
\end{aligned}
$$


for $|x|$ sufficiently large. That is for $\varepsilon>0$ sufficiently small, (3.33) holds. If $\|u\|_{\mu_{1}} \leq$ $e^{-\sqrt{\mu_{2}}|x|}$, then

$$
\left.\Phi(\mathcal{U}) \geq 2 c_{1}+c_{2}-\frac{\beta \gamma^{2}}{8} C_{1} e^{-2 \sqrt{\mu_{2}}|x|}-C_{2} e^{-\left(\sqrt{\mu_{1}}+\sqrt{\mu_{2}}\right)|x|}\right)-k_{2} e^{-2\left(\sqrt{\mu_{1}}-\rho\right)|x|} .
$$

Then recalling that $\sqrt{\mu_{2}}<\sqrt{\mu_{1}}-2 \rho$, we find (3.33) holds for $\varepsilon>0$ sufficiently small. This completes the proof.

Now for $x \in \mathbb{R}^{3} \backslash\{0\}$, we define a class $\Gamma_{2}(x) \subset C\left([0,1], \mathcal{M}_{2}\right)$ by

$$
\Gamma_{2}(x)=\left\{p \in C\left([0,1], \mathcal{M}_{2}\right): p(0)=\mathcal{N}\left(U_{1}, U_{2}\right), p(1)=\mathcal{N}\left(U_{1, x}+U_{1,-x}, U_{2}\right)\right\}
$$

and put

$$
c_{2}(x)=\inf _{p \in \Gamma_{2}(x)} \sup _{t \in[0,1]} \Phi(p(t)) .
$$

We also note that from the definitions of $\mathcal{N}$ and $\Phi$, we have that $\mathcal{N}\left(U_{1, x}+\right.$ $\left.U_{1,-x}, U_{2}\right)-\left(U_{1, x}+U_{1,-x}, U_{2}\right) \longrightarrow 0$ in $\mathbb{H}$ as $|x| \longrightarrow \infty$ and then

$$
\lim _{|x| \longrightarrow \infty} \Phi\left(\mathcal{N}\left(U_{1, x}+U_{1,-x}, U_{2}\right)\right)=2 I_{1}\left(U_{1}\right)+I_{2}\left(U_{2}\right)=2 c_{1}+c_{2} .
$$

We can now finish the proof of Theorem 1 for $i=2$.

Proof of Theorem 1 . Let $\varepsilon \in\left(0, \delta_{2} / 2\right)$ sufficiently small. Let $\beta \in\left(\beta_{\varepsilon}, 0\right)$. To complete the proof, it is sufficient to show that there exists $\delta>0$ and $R>0$ such that

$$
2 c_{1}+c_{2}+\delta<c_{2}(x)<2 c_{1}+c_{2}+\delta_{2} / 2 \text { for }|x|>R .
$$

In fact, if the inequalities above hold, we have by (3.34) that we can choose $x \in \mathbb{R}^{3}$ such that $|x|>R$ and

$$
\Phi\left(\mathcal{N}\left(U_{1, x}+U_{1,-x}, U_{2}\right)\right)<2 c_{1}+c_{2}+\delta .
$$

That is $\Phi(p(1))<c_{2}(x)$ for all $p \in \Gamma_{2}(x)$. We also have $\Phi(p(0))<\frac{7}{4} c_{1}+c_{2}$. Then since the Palais-Smail condition holds by (2) of Lemma 4 on $\Phi^{\left(2 c_{1}+c_{2}, 2 c_{1}+c_{2}+\delta_{2} / 2\right)}$, we have by a standard mountain pass argument that there exists a critical point $\mathcal{U}$ of $\Phi$ with $\Phi(\mathcal{U})=c_{2}(x)$.

From the definition of $\varepsilon$ and Lemma 5 , one can see the pass $p \in \Gamma_{2}(x)$ defined by

$$
p(s)=\mathcal{N}\left(U_{1, s x}+U_{1,-s x}, U_{2}\right), \quad s \in[0,1]
$$

satisfies $\max _{s \in[0,1]} \Phi(p(s)) \leq 2 c_{1}+c_{2}+\varepsilon$. Then the second inequality of (3.35) holds. We now show that the first inequality of (3.35) holds. We first see that there exists $\bar{R}>2 R_{\varepsilon}$ such that,

$$
\begin{aligned}
& b_{R_{\varepsilon}}(U) \geq 8 c_{1}-\frac{1}{2} \min \left\{\frac{1}{2 m_{4}^{4}}, c_{1}\right\} \\
& \text { for } \mathcal{U}=(U, V) \in \Lambda_{2, \varepsilon}\left(R_{\varepsilon}\right) \text { with } \quad \eta(U) \geq \bar{R},
\end{aligned}
$$


where $\eta$ is the function defined by (3.15). By Proposition 1 , each $\mathcal{U}=(U, V) \in$ $\Lambda_{2, \varepsilon}\left(R_{\varepsilon}\right)$ has the form

$$
\mathcal{U}=\left(\alpha\left(U_{1, x}+U_{1,-x}\right)+u, \gamma U_{2}+v\right)
$$

with $\alpha \in\left(1-\alpha_{\varepsilon}, 1+\alpha_{\varepsilon}\right), \gamma \in\left(1-\gamma_{\varepsilon}, 1+\gamma_{\varepsilon}\right)$ and $(u, v) \in\left\{U_{1, x}, U_{1,-x}\right\}^{\perp} \times\left\{U_{2}\right\}^{\perp}$ with $\|u\|_{\mu_{1}}^{2}+\|v\|_{\mu_{2}}^{2} \leq \delta_{\varepsilon}$. Since $\lim _{\varepsilon \longrightarrow 0} \delta_{\varepsilon}=\lim _{\varepsilon \longrightarrow 0} \alpha_{\varepsilon}=0$, we may assume that $\varepsilon>0$ is sufficiently small that

$$
8 \alpha_{\varepsilon}^{2} c_{1}-\delta_{\varepsilon}>8 c_{1}-\frac{1}{2} \min \left\{\frac{1}{2 m_{4}^{4}}, c_{1}\right\} .
$$

Since $\mathcal{U}$ has the form (3.37),

$$
\begin{aligned}
b_{R_{\varepsilon}}(U) & =\int_{\mathbb{R}^{3} \backslash B_{R_{\varepsilon}}(0)}|U|_{\mu_{1}}^{2} \\
& =\left\|\alpha\left(U_{1, x}+U_{1,-x}\right)+u\right\|_{\mu_{1}}^{2}-\int_{B_{R_{\varepsilon}}(0)}|U|_{\mu_{1}}^{2} \\
& \geq \alpha^{2}\left\|U_{1, x}+U_{1,-x}\right\|_{\mu_{1}}^{2}-\|u\|_{\mu_{1}}^{2}-2 \int_{B_{R_{\varepsilon}}(0)}\left|U_{1, x}+U_{1,-x}\right|_{\mu_{1}}^{2}
\end{aligned}
$$

holds. Then noting that

$$
\left\|U_{1, x}+U_{1,-x}\right\|_{\mu_{1}}^{2} \longrightarrow 8 c_{1} \quad \text { and } \quad \int_{B_{R_{\varepsilon}}(0)}\left|U_{1, x}+U_{1,-x}\right|_{\mu_{1}}^{2} \longrightarrow 0, \quad \text { as }|x| \longrightarrow \infty,
$$

we find by (3.38) that there exists $\bar{R}$ such that for each $\mathcal{U}=(U, V) \in \Lambda_{2, \varepsilon}\left(R_{\varepsilon}\right)$ with $\eta(\mathcal{U}) \geq \bar{R},(3.36)$ holds. Now we choose $x \in \mathbb{R}^{3}$ so large that $|x|>\bar{R}$. Then

$$
b_{R_{\varepsilon}}\left(\mathcal{N}_{1}\left(U_{1, x}+U_{1,-x}\right)\right) \geq 8 c_{1}-\frac{1}{2} \min \left\{\frac{1}{2 m_{4}^{4}}, c_{1}\right\} .
$$

Let $p=\left(p_{1}, p_{2}\right) \in \Gamma_{2}(x)$ such that $\sup _{t \in[0,1]} \Phi(p(t)) \leq 2 c_{1}+c_{2}+\varepsilon$. From the definition,

$\eta\left(p_{1}(1)\right)=\eta\left(\mathcal{N}_{1}\left(U_{1, x}+U_{1,-x}\right)\right)>\bar{R} \quad$ and $\quad b_{R_{\varepsilon}}\left(p_{1}(1)\right) \geq 8 c_{1}-\frac{1}{2} \min \left\{\frac{1}{2 m_{4}^{4}}, c_{1}\right\}$.

On the other hand, recalling that $\Phi_{2}(\mathcal{U}) \geq c_{2}$, we have by (2.11) that $\Phi_{1}(\mathcal{U}) \leq \frac{7}{4} c_{1}$. Then by the definition of $\varepsilon, b_{R_{\varepsilon}}\left(p_{1}(0)\right)<7 c_{1} \leq 8 c_{1}-\min \left\{\frac{1}{2 m_{4}^{4}}, c_{1}\right\}$, there exists $t \in(0,1)$ such that $b_{R_{\varepsilon}}\left(p_{1}(t)\right)=8 c_{1}-\min \left\{\frac{1}{2 m_{4}^{4}}, c_{1}\right\}$. Then by $(3.36), \eta\left(p_{1}(t)\right)<\bar{R}$. Therefore by the continuity of $\eta$, we have that there exists $t_{0} \in(0, t)$ such that $\eta\left(p_{1}\left(t_{0}\right)\right)=\bar{R}$. By Proposition 2, we have

$$
\Phi\left(p\left(t_{0}\right)\right) \geq 2 c_{1}+c_{2}+\beta M_{0} e^{-2 \sqrt{\mu_{2}} \bar{R}} .
$$

Therefore we obtain that $\sup _{t \in[0,1]} \Phi(p(t))>2 c_{1}+c_{2}+\beta M_{0} e^{-2 \sqrt{\mu_{2}} \bar{R}}$. Thus by the mountain pass theorem (cf. [17]), we find that there exists a critical point $\mathcal{U}$ of $\Phi$ with $\Phi(\mathcal{U})=c_{2}(x)$. 


\section{Multiple existence}

In the previous section, we proved Theorem 1 in the case that $i=2$. The proofs for the cases $i \in\{4,6,8,12,20\}$ are slight modifications of the proof for $i=2$. We give a sketch of the proof for $i=8$. Let $k \geq 1$ and $S^{(k)}=\left(1, \sigma_{k}, \sigma_{k}^{2}, \ldots, \sigma_{k}^{k-1}\right)$ be a finite group acting on $\mathbb{R}^{3}$ defined by

$$
\sigma_{k} x=\left(\left(\cos \frac{2 \pi}{k}\right) x_{1}-\left(\sin \frac{2 \pi}{k}\right) x_{2},\left(\sin \frac{2 \pi}{k}\right) x_{1}+\left(\cos \frac{2 \pi}{k}\right) x_{2}, x_{3}\right)
$$

for $x=\left(x_{1}, x_{2}, x_{3}\right) \in \mathbb{R}^{3}$.

We also define a mapping $\kappa: \mathbb{R}^{3} \longrightarrow \mathbb{R}^{3}$ by

$$
\kappa x=\left(x_{1}, x_{2},-x_{3}\right) \quad \text { for } \quad x=\left(x_{1}, x_{2}, x_{3}\right) \in \mathbb{R}^{3} .
$$

We put

$$
\widetilde{H}_{k}=\left\{u \in H: u(\sigma x)=u(x) \text { and } u(\kappa x)=u(x) \text { for } x \in \mathbb{R}^{3} \text { and } \sigma \in S^{(k)}\right\},
$$

and $\widetilde{\mathbb{H}}_{k}=\widetilde{H}_{k} \times \widetilde{H}_{k}$. By the equivariance of $\Phi$ under the actions $\sigma$ and $\kappa$, we have that each critical point $u$ of $\Phi$ in $\mathbb{H}_{k}$ is a critical point of $\Phi$ in $\mathbb{H}$. We denote by $G$ the finite group action on $\mathbb{R}^{3}$ which is generated by $S^{(4)}$ and $\tau$. To prove the case that $i=8$, we use, instead of $b_{r}$, the function $b_{r}^{(8)}$ which is defined by

$$
b_{r}^{(8)}(U)=\int_{\mathbb{R}^{3} \backslash A_{r}}|U|_{\mu_{1}}^{2} \quad \text { for } \quad r>0 \quad \text { and } \quad U \in H .
$$

where $D_{r}=\left\{x=\left(x_{1}, x_{2}, x_{3}\right) \in \mathbb{R}^{3}: x_{1}^{2}+x_{2}^{2}<r\right\}$ and

$$
A_{r}=D_{r} \cup\left\{x=\left(x_{1}, x_{2}, x_{3}\right) \in \mathbb{R}^{3}:\left|x_{3}\right|<r\right\} .
$$

We put $\mathcal{M}_{8}=\mathcal{M}_{+} \cap \widetilde{\mathbb{H}}_{4}$ and

$$
\Lambda_{8, \varepsilon}(R)=\left\{\mathcal{U}=(U, V) \in \Phi^{8 c_{1}+c_{2}+\varepsilon} \cap \mathcal{M}_{8}: b_{R}^{(8)}(U)>32 c_{1}-\min \left\{\frac{1}{2 m_{4}^{4}}, c_{1}\right\}\right\}
$$

for $(\varepsilon, R) \in \mathbb{R}^{+} \times \mathbb{R}^{+}$. We also choose $\delta_{8}>0$ so small that

$$
c_{1}+k c_{2} \notin\left[8 c_{1}+c_{2}-\delta_{8}, 8 c_{1}+c_{2}+\delta_{8}\right] \text { for all } k \in \mathbb{N} .
$$

Then we have following lemmas and propositions instead of Lemma 4, Lemma 5 , Proposition 1 and Proposition 2.

Lemma 8. (1) There exists $\beta_{1}^{(8)} \in(-1,0)$ such that for each critical point $\mathcal{U} \in$ $\mathcal{M}_{8} \cap \Phi^{8 c_{1}+2 c_{2}}$

$$
\Phi(\mathcal{U}) \in \cup_{i \geq 1, j \geq 1}\left[i c_{1}+j c_{2}-\delta_{8} / 2, i c_{1}+j c_{2}+\delta_{8} / 2\right]:
$$

(2) Let $\beta \in\left(\beta_{1}^{(8)}, 0\right)$ and $\left\{\mathcal{U}_{n}\right\} \subset \mathcal{M}_{8}$ such that

$$
\lim _{n \longrightarrow \infty} \nabla \Phi\left(\mathcal{U}_{n}\right) \longrightarrow 0 \quad \text { and } \quad \lim _{n \longrightarrow \infty} \Phi\left(\mathcal{U}_{n}\right)=8 c_{1}+c_{2}+\varepsilon
$$

with $\varepsilon \in\left(0, \delta_{8} / 2\right)$. Then there exists a convergence subsequence $\left\{\mathcal{U}_{n_{i}}\right\} \subset\left\{\mathcal{U}_{n}\right\}$. 
The proof of Lemma 8 is the same as that of Lemma 4 . Now we fix $\beta \in$ $\left(\beta_{1}^{(8)}, 0\right)$, Then we have the following two propositions.

Proposition 3. For $\varepsilon>0$ sufficiently small, there exists $\left(R_{\varepsilon}^{(8)}, \delta_{\varepsilon}^{(8)}, \alpha_{\varepsilon}^{(8)}, \gamma_{\varepsilon}^{(8)}\right) \in$ $\left(\mathbb{R}^{+}\right)^{4}$ such that $\lim _{\varepsilon \longrightarrow 0} \delta_{\varepsilon}^{(8)}=\lim _{\varepsilon \longrightarrow 0} \alpha_{\varepsilon}^{(8)}=\lim _{\varepsilon \longrightarrow 0} \gamma_{\varepsilon}^{(8)}=0$ and each $\mathcal{U} \in$ $\Lambda_{8, \varepsilon}\left(R_{\varepsilon}^{(8)}\right)$ has the form

$$
\mathcal{U}=\left(\alpha \sum_{i=1}^{8} U_{1, x_{i}}+u, \gamma U_{2,0}+v\right)
$$

where $\alpha \in\left(1-\alpha_{\varepsilon}^{(8)}, 1+\alpha_{\varepsilon}^{(8)}\right), \gamma \in\left(1-\gamma_{\varepsilon}^{(8)}, 1+\gamma_{\varepsilon}^{(8)}\right),\left\{x_{i}\right\}_{i=1}^{8} \subset \mathbb{R}^{3}$ forms a octahedron,

$$
\begin{gathered}
\left|x_{i}\right| \geq R_{\varepsilon}^{(8)} \quad i=1, \ldots, 8, \quad x=\mathcal{B}\left(\left.U\right|_{B_{R_{0}}(x)}\right) \\
\widehat{U}(z)<\frac{1}{2}|\widehat{U}|_{\infty} \quad \text { for } \quad z \in \mathbb{R}^{3} \backslash\left(\bigcup_{i=1}^{8} B_{R_{0}}\left(x_{i}\right)\right)
\end{gathered}
$$

and

$$
(u, v) \in\left\{U_{1, x_{i}}: 1 \leq i \leq 8\right\}^{\perp} \times\left\{U_{2}\right\}^{\perp} \quad \text { with } \quad\|u\|^{2}+\|v\|^{2} \leq \delta_{\varepsilon}^{(8)} .
$$

Sketch of proof. For $r>0$, we put

$$
B_{r, I d}=A_{r+1} \cup\left\{x=\left(x_{1}, x_{2}, x_{3}\right) \in \mathbb{R}^{3}: \min _{i=1,2}\left|x_{i}\right|<1\right\} \backslash \overline{A_{r}}
$$

and

$$
B_{r, g}=g\left(B_{r, I d}\right) \text { for all } g \in G .
$$

Let $\varphi_{r, I d} \in C^{\infty}\left(\mathbb{R}^{3} ;[0,1]\right)$ such that

$$
\varphi_{r, I d}=\left\{\begin{array}{lll}
1 & \text { on } & \mathbb{R}^{3} \backslash B_{r, I d} \\
0 & \text { on } & \partial B_{r+\frac{1}{2}, I d} \cup\left\{x \in \mathbb{R}^{3} \backslash B_{r+\frac{1}{2}, I d} ; \min _{i=1,2}\left|x_{i}\right|=0\right\},
\end{array}\right.
$$

and $\varphi_{r, g}(x)=\varphi_{r, I d}(g x)$ for $x \in \mathbb{R}^{3}$ and $g \in G$. For $R>r+\frac{1}{2}$ and $\mathcal{U}=(U, V) \in$ $\Lambda_{8, \varepsilon}\left(R_{\varepsilon}^{(8)}\right)$, we put

$$
\varphi_{r, g} U=W_{8,0}+\sum_{i=1}^{8} W_{8, i}
$$

where supp $W_{8, i}$ are mutually disjoint and for $1 \leq i, j \leq 8, W_{8, i+1}=\sigma_{4}\left(W_{8, i}\right)$ for $1 \leq i \leq 7, i \neq 4$ and $W_{8, i+4}=\kappa W_{8, i}$ for $1 \leq i \leq 4$. Then by using $B_{r, g}, \varphi_{r, g}$ and $W_{8, i}$ instead of $B_{r, z}, \varphi_{r, z}$ and $W_{ \pm}$, we can prove the assertion by the same argument as in the proof of Proposition 1.

Proposition 4. There exists $M_{0}^{(8)}>0$ such that for $\varepsilon$ sufficiently large,

$$
\Phi(\mathcal{U}) \geq 8 c_{1}+c_{2}-\beta M_{0}^{(8)} e^{-2 \sqrt{\mu_{2}}|x|} \quad \text { for } \quad \mathcal{U} \in \Lambda_{8, \varepsilon}\left(R_{\varepsilon}^{(8)}\right),
$$

where $x \in \mathbb{R}^{3}$ such that $\mathcal{U}$ satisfies (4.2). 
Proof. As in Lemma 6, we have that there exists $k_{1}^{(8)}>0$ such that for $\varepsilon>0$ sufficiently small, each $\mathcal{U}=(U, V) \in \mathcal{M}_{8} \cap \Phi^{8 c_{1}+c_{2}+\varepsilon}$ satisfies

$$
\Phi_{2}(\mathcal{U}) \geq c_{2}+k_{1}^{(8)}\|v\|_{\mu_{1}}^{2}
$$

where $v \in\left\{U_{2}\right\}^{\perp}$ such that $V=\alpha U_{2}+v$ for some $\alpha \in \mathbb{R}$. The proof of Lemma 6 is valid for this case. Next for $r>0$ and $x \in \partial B_{1}(0)$, we put

$$
\begin{aligned}
\Omega_{0}(r) & =\left\{z=\left(z_{1}, z_{2}, z_{3}\right) \in \mathbb{R}^{3}: \min _{i=1,2,3}\left|z_{i}\right| \leq r\right\}, \\
\Omega_{1}(r)= & \left\{z \in \mathbb{R}^{3} \backslash \Omega_{0}(r): z_{1}, z_{2}, z_{3} \geq 0\right\}, \\
\Omega_{i, h}(r)= & h\left(\Omega_{i}(r)\right)=\left\{h z: z \in \Omega_{i}\right\}, \text { for } i=0,1, \\
& h \in G_{8}=\left\{1, \sigma_{4}, \sigma_{4}^{2}, \sigma_{4}^{3}, \kappa, \kappa \sigma_{4}, \kappa \sigma_{4}^{2}, \kappa \sigma_{4}^{3}\right\},
\end{aligned}
$$

and

$$
a_{n, h}(u)=\int_{\Omega_{0, h}(n)}|u|_{\mu_{1}}^{2} d x \quad \text { for each } n \geq 1, h \in G_{8} \quad \text { and } \quad u \in H .
$$

For $n \geq 1$ and $h \in G_{8}$, we define $\psi_{n, h} \in C^{\infty}\left(\mathbb{R}^{3},[0,1]\right)$ such that

$$
\psi_{n, h}(z)=1 \quad \text { on } \quad \Omega_{1, h}(n+1) \quad \text { and } \quad \psi_{n, h}(z)=0 \quad \text { on } \quad \mathbb{R}^{3} \backslash \Omega_{1, h}(n) .
$$

Then using $\Omega_{i, h}(r)$ and $\psi_{n, h}(z)$ instead of $\Omega_{0}(r, x), \Omega_{ \pm}(r, z)$ and $\psi_{n, x}^{ \pm}$, we can see that all the computations in proof of Lemma 7 for $U_{1, x}+\psi_{n, x}^{+}\left(U_{1,-}+u\right)$ are valid for $U_{1, x_{1}}+\psi_{n, I d}\left(\sum_{i=2}^{8} U_{1, x_{i}}+u\right)$. That is we obtain by a parallel argument as in the proof of Lemma 7 that for each $\mathcal{U} \in \Lambda_{8, \varepsilon}\left(R_{\varepsilon}^{(8)}\right)$,

$$
\Phi_{1}(\mathcal{U}) \geq 32 c_{1}-\frac{1}{4} \int_{\mathbb{R}^{3}} \beta V^{2} U^{2}+O\left(e^{-2\left(\sqrt{\mu_{1}}-\rho\right)(|x|)}\right)
$$

where $x \in \mathbb{R}^{3}$ such that $\mathcal{U}$ has the form (4.2). Then the assertion follows again by the same argument as in the proof of Proposition 2.

Here we define a class $\Gamma_{8}(x), x=\left(x_{1}, x_{1}, x_{1}\right) \in \mathbb{R}^{3} \backslash\{0\}$ by

$$
\Gamma_{8}(x)=\left\{p \in C\left([0,1], \mathcal{M}_{2}\right): p(1)=\mathcal{N}\left(\sum_{h \in G} U_{1, h x}, U_{2}\right), p(0)=\mathcal{N}\left(U_{1}, U_{2}\right)\right\} .
$$

Then by the same argument as in the proof of Lemma 5, we have that

Lemma 9. For given $\varepsilon>0$, there exists $\beta_{\varepsilon}^{(8)} \in\left(\beta_{1}^{(8)}, 0\right)$ such that for each $\beta \in$ $\left(\beta_{\varepsilon}^{(8)}, 0\right)$ and for each $x=\left(x_{1}, x_{1}, x_{1}\right) \in \mathbb{R}^{3} \backslash\{0\}$, there exists a path $p \in \Gamma_{8}(x)$ such that

$$
\max _{t \in[0,1]} \Phi(p(t)) \leq 8 c_{1}+c_{2}+\varepsilon
$$


Then the proof of Theorem 1 for $i=8$ carried out by the same way as that for the case $i=2$, using Proposition 3, Proposition 4 and Lemma 9.

Theorem 2 is proved by the same argument for Theorem 1 . We just need a minor modification of the setting. Let $k \geq 1$ and put

$$
\widetilde{b}_{r}(U)=\int_{\mathbb{R}^{3} \backslash D_{r}}|U|_{\mu_{1}}^{2} \quad \text { for } \quad r>0 \quad \text { and } \quad U \in H .
$$

and

$$
\widetilde{\Lambda}_{k, \varepsilon}(R)=\left\{\mathcal{U}=(U, V) \in \Phi^{2 c_{1}+c_{2}+\varepsilon} \cap \mathcal{M}_{k}: \widetilde{b}_{R}(U)>k c_{1}-\min \left\{\frac{1}{2 m_{4}^{4}}, c_{1}\right\}\right\}
$$

for $R, \varepsilon>0$. Then by using $\widetilde{b}_{R}$ instead of $b_{R}$, we have that for $\varepsilon>0$ sufficiently small, there exists $\widetilde{R}_{\varepsilon}^{(k)}>0$ such that each $\mathcal{U} \in \widetilde{\Lambda}_{k, \varepsilon}\left(\widetilde{R}_{\varepsilon}^{(k)}\right)$ has the form

$$
\mathcal{U}=\left(\alpha \sum_{i=0}^{k-1} U_{1, \sigma_{k}^{i} x}+u, \beta U_{2}+v\right)
$$

where $\alpha$ and $\beta$ are close to $1, u, v$ are small. From the definition of the space $\widetilde{H}_{k}$ and $\widetilde{b}_{r}$, one can see that

$$
x \in\left\{\left(x_{1}, x_{2}, 0\right) \in \mathbb{R}^{3}: x_{1}, x_{2} \in \mathbb{R}\right\} \quad \text { with } \quad|x| \geq R_{\varepsilon} .
$$

We also have, instead of Proposition 2, that there exists $\widetilde{\beta}_{1}^{(k)} \in(-1,0)$ and $\widetilde{M}_{0}>0$ satisfying that for $\beta \in\left(\widetilde{\beta}_{1}^{(k)}, 0\right)$ and for $\varepsilon>0$ sufficiently small,

$$
\Phi(\mathcal{U}) \geq k c_{1}+c_{2}-\beta \widetilde{M}_{0}^{(k)} e^{-2 \sqrt{\mu_{1}}|x|} \quad \text { for each } \mathcal{U} \in \widetilde{\Lambda}_{k, \varepsilon}\left(\widetilde{R}_{\varepsilon}^{(k)}\right)
$$

where $x \in \mathbb{R}^{3}$ such that $\mathcal{U}$ has the form (3.1).

Then the assertion of Theorem 2 follows by the same argument as in the proof of Theorem 1 for the case that $i=2$.

\section{References}

[1] R. A. Adams, Sobolev spaces, Academic Press, New York, 1975.

[2] N. Akhmediev and A. Ankiewicz, Partially coherent solitons on a finite background, 'jus. Rev. Lett 82 (1999), 2661-2664.

[3] A. Ambrosetti and E. Colorado, Bounded and ground states of coupled nonlinear schrodinger equations, preprint.

[4] T. Bartsch and Tobias Weth, Three nodal solutions of singularly perturbed elliptic equatuions on domanis without topology, Ann. I. H. Poincare 22 (2005), 259-281.

[5] G. Cerami \& D. Passaseo, The effect of concentrating potentials in some singulerly perturbed problems, Carc. Var. PDE. 17 (2003), 257-281.

[6] B. D. Esry, C. H. Greene, J. P. Burke, and J. L. Bohn, Jartree-fock theory for double condensates, Phys. Rev. Lett 78 (1997), 3494-3497.

[7] B. Gidas, W. M. Ni, and L. Nirenberg, Symmetry of positive solutions fo nonlinear elliptic equation in $r^{n}$, Adv. Math. Suppl. Stud. 7 (1981), 369-402. 
[8] N. Hirano and N. Shioji, Multiple existence of solutions for a coupled nonlinear schrodinger equations, Nonlinear Analysis TMA, to appear.

[9] M. K. Kwong, Uniqueness of positive solutions of $-\delta u-u+u^{p}=0$ in $r^{n}$, Arch. Rat. Mech. Anal. 105 (1989), 243-266.

[10] T. C. Lin and J. Wei, Ground state of $n$ coupled nonlinear Schrodinger equations in $r^{n}$, Communications in Mathematical Physics 255 (2005), 629-653.

[11] T. C. Lin and J. Wei, Spikes in two-component systems of nonlinear Schrodinger equations, Annales de l'Institut H. Poincare 22 (2005), 403-439.

[12] T. C. Lin and J. Wei, Solitary and self-similar solutions of two-component system of nonlinear Schrodinger equations, Phys. D 2 (2006), 99-115.

[13] P.L. Lions, The concentration-compactness principle in the calculus of variations. the locally compact case. part 1, Ann. Inst. H. Poincare 1 (1984), 109-145.

[14] L.A. Maia, E. Montefusco, and B. Pellacci, Positive solutions for a weakly coupled nonlinear Schrodinger system, preprint.

[15] C.R. Menyuk, Nonlinear pulse propagation in birefrignent optical fibers, IEEE J. Quantum Electron 23 (1987), 174-176.

[16] A. Pomponio, Coupled nonlinear Schrodinger systems with potentials, J. Diff. Equations 227 (2006), 258-281.

[17] P. Rabinowitz, Minimax methods in critical point theory with applications to differential equations, C. B. M. S. Regional Conf. Math. Series 65. A.M.S. Providence, 1986.

[18] S. Solimini \& M. Struwe G. Cerami, Some existence results for superlinear elliptic boundary value problems involving critical exponents, J. Func. Anal. 69 (1986), 289306.

[19] W. C. Troy, Symmetry properties in systems of semilinear elliptic equations, J. Diff. Equations 42 (1981), 400-413.

[20] J. Wei, On the construction of single-peaked solutions to a singularly perturbed semilinear dirichlet problem, J. Diff. Equations 129 (1996), 315-333.

[21] J. Wei and T. Weth, Existence of nonraidal symmetric bound states for a system of coupled Schrodinger equations, preprint.

[22] X. Zhu, A perturbation result on positive entire solutions of a semilinear elliptic equation, J. Diff. Equations 92 (1991), 163-178.

Norimichi Hirano

Department of Mathematics

Graduate School of Enviornment and Information Sciences

Yokohama National University

Tokiwadai, Hodogayaku

Yokohama

Japan

e-mail: hirano@math.sci.ynu.ac.jp

Received: 28 September 2007.

Revised: 21 March 2008.

Accepted: 25 April 2008. 